\title{
Can we find steady-state solutions to multiscale rarefied gas flows within dozens of iterations?
}

\author{
Wei Su ${ }^{\mathrm{a}, 1}$, Lianhua Zhu ${ }^{\mathrm{a}, 1}$, Peng Wang ${ }^{\mathrm{a}}$, Yonghao Zhang ${ }^{\mathrm{a}}$, Lei $\mathrm{Wu}^{\mathrm{a}, \mathrm{b}, *}$ \\ ${ }^{a}$ James Weir Fluids Laboratory, Department of Mechanical and Aerospace Engineering, University of \\ Strathclyde, Glasgow G1 1XJ, UK \\ ${ }^{b}$ Department of Mechanics and Aerospace Engineering, Southern University of Science and Technology, \\ Shenzhen 518055, China
}

\begin{abstract}
One of the central problems in the study of rarefied gas dynamics is to find the steady-state solution of the Boltzmann equation quickly. When the Knudsen number is large, i.e. the system is highly rarefied, the conventional iterative scheme can lead to convergence within a few iterations. However, when the Knudsen number is small, i.e. the flow falls in the nearcontinuum regime, hundreds of thousands iterations are needed, and yet the "converged" solutions are prone to be contaminated by accumulated error and large numerical dissipation. Recently, based on the gas kinetic models, the implicit unified gas kinetic scheme (UGKS) and its variants have significantly reduced the iterations in the near-continuum flow regime, but still much higher than that of the highly rarefied gas flows. In this paper, we put forward a general synthetic iterative scheme (GSIS) to find the steady-state solutions of general rarefied gas flows within dozens of iterations at any Knudsen number. The key ingredient of our scheme is that the macroscopic equations, which are solved together with the Boltzmann equation and help to adjust the velocity distribution function, not only asymptotically preserve the Navier-Stokes limit in the framework of Chapman-Enskog expansion, but also contain the Newton's law for stress and the Fourier's law for heat conduction explicitly. For this reason, like the implicit UGKS, the constraint that spatial cell size should be smaller than the mean free path of gas molecules is removed, but we do not need the complex evaluation of numerical flux at cell interfaces. What's more, as the GSIS does not rely on the specific kinetic model/collision operator, it can be naturally extended to quickly find converged solutions for mixture flows and even flows involving chemical reactions. These two superior advantages are expected to accelerate the slow convergence in simulation of near-continuum flows via the direct simulation Monte Carlo method and its low-variance version.
\end{abstract}

\footnotetext{
*Corresponding author:

Email address: wul@sustech.edu.cn (Lei Wu)

${ }^{1}$ Both authors contributed equally.
} 


\section{Introduction}

Multiscale rarefied gas flows involving a wide range of Knudsen number have been encountered in massive engineering problems, e.g. high-altitude aerothermodynamics of space vehicles, microelectromechanical systems, and gas transportation in ultra-tight shale strata. A gas flow can be described by either the macroscopic or the mesoscopic model. At the macroscopic level, the gas is assumed as a continuous medium and the evolution of gas system is described in terms of the spatial and temporal variations of the familiar flow properties such as density, velocity, pressure and temperature. The mathematical description of any macroscopic model is grounded in two primary aspects: 1) the conservation laws that describe how the mass, momentum and energy should be conserved during transport processes, and 2) the constitutive equations that describe how the fluxes of mass dissipation, momentum diffusion and heat conduction response to various stimuli such as pressure difference, gradients of temperature and velocity, and external force. The Navier-Stokes equations provide the conventional mathematical model for a gas as a continuum, in which the conservation laws are closed by the famous constitutive equations of the Newton's law of viscosity and Fourier's law of heat conduction. Since the transport terms are expressed in terms of the first-order macroscopic quantities, the Navier-Stokes equations are only valid when the length scale of the gradients of the macroscopic variables is much larger than the mean free path of gas molecules, i.e. the Knudsen number is far smaller than one [1]. Many other higher-order macroscopic equations are proposed for larger Knudsen numbers, but none of them is able to describe the multiscale rarefied gas flows from the continuum to free molecular flow regimes [2].

The mesoscopic model postulates that the gas is not continuous but is composed of a finite number of molecules. The molecules rush hither and thither, and strike with boundary and collide with each other. Actually, the macroscopic transport phenomena stem no other than the random motion of gas molecules. The mathematical model at the mesoscopic level is the Boltzmann equation, which governs the evolution of the one-particle velocity distribution function providing information on the state of every molecule at all times [3]. Then, macroscopic flow properties are identified with average values of the molecular quantities. Note that the Boltzmann equation is applicable for the entire range of Knudsen number, as long as the gas is dilute, i.e. the molecular mean free path is much larger than the dimension of gas molecules.

The Boltzmann equation can be numerically solved either in discretized molecular velocity space via the discrete velocity method (DVM) [4], or by applying the direct simulation Monte Carlo (DSMC) method that uses a collection of particles to represent random points in the molecular velocity space [3]. Compared to the traditional computational fluid dynamic (CFD) techniques for solving macroscopic equations, the Boltzmann equation and its simplified kinetic model equations are much more expensive to solve in terms of computation time and memory. This is mainly due to the following facts. First of all, additional dimensions of the molecular velocity space are required to be discretized in DVM and particles are required to generate in DSMC. Second, since the random behaviors of gas molecules are modeled on length and time scales comparable to the cell size and simulation time interval, 
respectively, in order to suppress numerical diffusion errors it is suggested that the size of grid cell and time interval should be smaller than the mean free path and mean collision time of gas molecules, respectively [5]. As a consequence, the computational cost dramatically increases as the gas flow approaches the near-continuum regime. Finally, in DVM, the conventional iterative scheme (CIS) to find steady-state solution converges extreme slowly for flows with low Knudsen numbers, since the exchange of information (e.g. perturbation in the flow field) through molecular streaming becomes very inefficient when binary collisions dominate [6]. Worse still, the "converged" solutions are prone to be contaminated by numerical errors, e.g. the accumulated error from finite discrete molecular velocities [7] and error stemming from the evaluation of molecular collisions, say, by the projection method [8] and the fast spectral method [9]. In DSMC, the simulation time also increases significantly due to this inefficient information exchange process in near-continuum flows. Note that the unified gas-kinetic scheme (UGKS) [10, 11, 12, 13, 14] can remove the restrictions on cell size and time step by simultaneously handling free streaming and collision of gas molecules. However, as information exchanging relays on the evolution of velocity distribution function, UGKS still needs a large number of iterations to obtained steady-state solutions for near-continuum flows [15, 16].

There has been a tremendous growth of researches on multiscale hybrid numerical methods that combine multiple models defined at fundamentally different length and time scales within the same overall spatial and temporal domain. Specifically for the flow of interest, the continuum CFD methods are used in regions where the Navier-Stokes equations are valid, while methods based on gas kinetic theory are applied in regions where the continuum equations fail [17, 18, 19, 20, 21, 22, 23]. However, intrinsic difficulties arise when coupling the two different models. First, the mechanism for continuum breakdown is unclear and the criterion to determine where the continuum model is valid relies on empirical parameters that vary for different flow conditions [21. Second, the continuum-kinetic coupling is strictly required to lie in the region that can be accurately modeled by the Navier-Stokes equations, so that the Boltzmann equation is still employed in low-Knudsen-number regions. Therefore, the CIS still needs lots of iterations to converge to the steady-state solution; also, DSMC still needs small cell size and time step and hence large evolutionary steps to find converged solution.

In recent years, the synthetic iterative scheme (SIS), which is initially developed for radiation transport process [24], has been extended to achieve high efficiency and accuracy in DVM, in particular with fast convergence property across the whole gas flow regimes [25, 26]. In this scheme, the gas kinetic equation and macroscopic equations are solved simultaneously on the same grid in the entire domain. Since the velocity distribution function is guided by the macroscopic flow quantities solved from diffusion-type equation at each iterative step, information propagates accurately and fast even when Knudsen number is small. When the Knudsen number is small, the synthetic macroscopic equations asymptotically regress to the Navier-Stokes equations. On the other hand, the SIS also preserves accuracy in other flow regimes since the macroscopic equations contain high-order terms to take into account rarefaction effects. The SIS has been successfully applied to Poiseuille flow in channels of arbitrary shapes using the Bhatnagar-Gross-Krook (BGK) kinetic model for single-species 
monatomic gases [27], and flows of binary and ternary gas mixtures driven by local pressure, temperature and concentration gradients using the McCormak model [28, 29, 30, 31, 32. It has also been extended to solve the linearized Boltzmann equation (LBE), where the role of realistic intermolecular potential in Poiseuille, Couette and thermal transpiration flows has been analyzed [6, 33].

It is interesting to note that the similar idea of SIS has also been used in DSMC, that is, in addition to traditional DSMC, macroscopic variables are solved and updated according to macroscopic rules/equations. For instances, in the information preservation DSMC, the information velocity is introduced to compute macroscopic velocity and shear stress, with the aim of removing "the statistical fluctuation source inherent in the DSMC method that results from the randomness of the thermal velocity" [34, 35, 36], although the rule of updating the information velocity and/or other macroscopic variables is not exactly derived from the Boltzmann equation. On the other hand, the moment-guided DSMC is proposed to reduce the statistical error, where the density, velocity and temperature are updated by the five exact macroscopic equations from conservation laws, but with the pressure tensor and heat flux calculated from the DSMC [37]. Similar idea is adopted in neutral gas kinetics, where, in addition to the five macroscopic equations for density, velocity and temperature, consistency terms are introduced to ensure that, upon convergence, solutions from the low-order macroscopic equations will be the same as that from the kinetic equation [38]. Although fast convergence is realized, this, however, will cause problems since if the spatial cell size is not resolved the DVM solution of gas kinetic equation is contaminated by the large numerical dissipation, for instances, see Fig. 1(d) in Ref. [16] and Fig. 6 below.

In DVM, the SIS can not only asymptotically achieve the Navier-Stokes limit with fast convergence rate, but also preserve accuracy in high Knudsen number regimes. The critical point to develop this scheme is that the macroscopic equations must explicitly contain both the constitutive relations predicting the transport phenomena at the continuum level, as well as high-order terms taking into account rarefaction effects. To the author's awareness, in rarefied gas dynamics the SIS is still limited to simple flows such as the Poiseuille, Couette and thermal transpiration flows, where the flow velocity is perpendicular to the computational domain, we refer to [25] for an example. In this paper, we shall develop the general SIS (GSIS) with the aim to find the steady-state solution of general rarefied gas flow within dozens of iterations at any Knudsen number. For the first step, we will consider only linearized flows in this paper.

The remainder of the paper is organized as follows. In Section 2, the LBE is introduced. In Section 3, the GSIS for general rarefied gas flow is proposed. Numerical tests to assess efficiency and accuracy of the proposed scheme are presented for stationary and periodic oscillatory problems in Sections 4, 5 and 6. The paper closes with some finial comments and outlooks in Section 7 .

\section{The linearized Boltzmann equation}

In kinetic theory, the state of a gaseous system is described by the one-particle velocity distribution $f(t, \boldsymbol{x}, \boldsymbol{v})$. Evolution of the velocity distribution function to the independent 
variables, i.e. time $t$, spatial position $\boldsymbol{x}=\left(x_{1}, x_{2}, x_{3}\right)$ and molecular velocity $\boldsymbol{v}=\left(v_{1}, v_{2}, v_{3}\right)$, is governed by the Boltzmann equation [1]. When the system deviates slightly from the global equilibrium described by

$$
f_{\text {eq }}(\boldsymbol{v})=\pi^{-3 / 2} \exp \left(-|\boldsymbol{v}|^{2}\right),
$$

the velocity distribution function of gas molecules can be linearized around $f_{\text {eq }}$ as:

$$
f(t, \boldsymbol{x}, \boldsymbol{v})=f_{\mathrm{eq}}(\boldsymbol{v})+\alpha h(t, \boldsymbol{x}, \boldsymbol{v}),
$$

where $\alpha h(t, \boldsymbol{x}, \boldsymbol{v})$ is the small perturbance satisfying $\left|\alpha h / f_{\text {eq }}\right| \ll 1$ with $\alpha$ being a small constant related to the amplitude of perturbation. The velocity distribution function $h(t, \boldsymbol{x}, \boldsymbol{v})$, however, is not necessary smaller than the equilibrium distribution function $f_{\text {eq }}$. The LBE for $h(t, \boldsymbol{x}, \boldsymbol{v})$ is:

$$
\frac{\partial h}{\partial t}+\boldsymbol{v} \cdot \frac{\partial h}{\partial \boldsymbol{x}}=L\left(h, f_{\mathrm{eq}}\right),
$$

where the linearized Boltzmann collision operator is [39]:

$$
L=\underbrace{\iint B(\theta,|\boldsymbol{u}|)\left[f_{\mathrm{eq}}\left(\boldsymbol{v}^{\prime}\right) h\left(\boldsymbol{v}_{*}^{\prime}\right)+f_{\mathrm{eq}}\left(\boldsymbol{v}_{*}^{\prime}\right) h\left(\boldsymbol{v}^{\prime}\right)-f_{\mathrm{eq}}(\boldsymbol{v}) h\left(\boldsymbol{v}_{*}\right)\right] \mathrm{d} \Omega \mathrm{d} \boldsymbol{v}_{*}}_{L^{+}}-\nu_{\mathrm{eq}}(\boldsymbol{v}) h(\boldsymbol{v}),
$$

and the equilibrium collision frequency is

$$
\nu_{\mathrm{eq}}(\boldsymbol{v})=\iint B(|\boldsymbol{u}|, \theta) f_{\mathrm{eq}}\left(\boldsymbol{v}_{*}\right) \mathrm{d} \Omega \mathrm{d} \boldsymbol{v}_{*} .
$$

Note that the relative velocity of the two molecules before binary collision is $\boldsymbol{u}=\boldsymbol{v}-\boldsymbol{v}_{*}$, and $\Omega$ is a unit vector along the relative post-collision velocity $\boldsymbol{v}^{\prime}-\boldsymbol{v}_{*}^{\prime}$. The deflection angle $\theta$ between the pre- and post-collision relative velocities satisfies $\cos \theta=\Omega \cdot \boldsymbol{u} /|\boldsymbol{u}|, 0 \leq \theta \leq \pi$. Finally, $B(\theta,|\boldsymbol{u}|)=|\boldsymbol{u}| \sigma$ is the collision kernel, with $\sigma$ being the differential cross-section that is determined by the intermolecular potential. In the present paper, we mainly consider the inverse power-law potentials, where the collision kernel is modeled as [39, 40]

$$
B(|\boldsymbol{u}|, \theta)=\frac{|\boldsymbol{u}|^{2(1-\omega)}}{K} \sin ^{\frac{1}{2}-\omega}\left(\frac{\theta}{2}\right) \cos ^{\frac{1}{2}-\omega}\left(\frac{\theta}{2}\right),
$$

with $\omega$ being the viscosity index (i.e. the shear viscosity $\mu$ of the gas is proportional to $T^{\omega}$ ) and $K$ some normalization constants [39]. HS and Maxwell molecules have $\omega=0.5$ and 1 , respectively. We will also consider the Lennard-Jones potential (the detailed implementation of which by the fast spectral method can be found in Ref. [41]) to demonstrate that the GSIS works for the LBE with general intermolecular potentials.

Note that we have presented the governing system in terms of dimensionless variables. The coordinate $\boldsymbol{x}$ is normalized by the characteristic flow length $H$, the molecular velocity $\boldsymbol{v}$ is normalized by the most probable speed $v_{\mathrm{m}}=\sqrt{2 k_{\mathrm{B}} T_{0} / m}$, the time $t$ is normalized by 
$H / v_{\mathrm{m}}$, and velocity distribution functions $f_{\text {eq }}$ and $h$ are normalized by $n_{0} / v_{\mathrm{m}}^{3}$, where $n_{0}$ is the average number density of the gas molecules, $T_{0}$ is the reference temperature, $k_{\mathrm{B}}$ is the Boltzmann constant, and $m$ is the mass of gas molecules.

To fully determine the gas dynamics in spatially-inhomogeneous problems, the gassurface boundary condition should be specified. In this paper, the Maxwell diffuse boundary condition is used: the velocity distribution function $f(t, \boldsymbol{x}, \boldsymbol{v})$ of the reflected gas molecules at the solid surface satisfies the following equation:

$$
f(t, \boldsymbol{x}, \boldsymbol{v})=\frac{2 \int_{v_{n}^{\prime}<0}\left|v_{n}^{\prime}\right| f\left(t, \boldsymbol{x}, \boldsymbol{v}^{\prime}\right) \mathrm{d} \boldsymbol{v}^{\prime}}{\pi T_{\mathrm{w}}^{2}} \exp \left(-\frac{\left|\boldsymbol{v}-\boldsymbol{U}_{\mathrm{w}}\right|^{2}}{T_{\mathrm{w}}}\right),
$$

where $T_{\mathrm{w}}$ is the wall temperature normalized by the reference temperature $T_{0}, \boldsymbol{U}_{\mathrm{w}}$ is the wall velocity normalized by the most probable speed $v_{\mathrm{m}}$, and $v_{n}$ is the normal component of the peculiar velocity $\boldsymbol{v}-\boldsymbol{U}_{\mathrm{w}}$ redirected into the gas.

The macroscopic quantities of interest including the number density $\rho$, bulk velocity $\boldsymbol{U}$, temperature $T$, pressure $p$, stress tensor $\sigma_{i j}$ and heat flux $\boldsymbol{q}$, which are further normalized by the dimensionless constant $\alpha$, can be calculated as

$$
\begin{array}{r}
\rho=\int h \mathrm{~d} \boldsymbol{v}, \quad \boldsymbol{U}=\int \boldsymbol{v} h \mathrm{~d} \boldsymbol{v}, \quad T=\frac{2}{3} \int|\boldsymbol{v}|^{2} h \mathrm{~d} \boldsymbol{v}-\rho, \quad p=\rho+T \\
\sigma_{i j}=2 \int\left(v_{i} v_{j}-\frac{|\boldsymbol{v}|^{2}}{3} \delta_{i j}\right) h \mathrm{~d} \boldsymbol{v}, \quad \boldsymbol{q}=\int \boldsymbol{v}|\boldsymbol{v}|^{2} h \mathrm{~d} \boldsymbol{v}-\frac{5}{2} \boldsymbol{U},
\end{array}
$$

where $\delta_{i j}$ is the Kronecker delta function, and $i, j=1,2,3$ represent the three orthogonal spatial directions in the Cartesian coordinates.

\section{The general synthetic iterative scheme}

The steady state solution of the integro-differential system (3) is usually solved by the CIS. Given the value of $h^{(k)}(\boldsymbol{x}, \boldsymbol{v})$ at the $k$-th iteration step, the velocity distribution function at the next iteration step is calculated by solving the following equation [40, 41, 42]:

$$
\nu_{\mathrm{eq}}(\boldsymbol{v}) h^{(k+1)}+\boldsymbol{v} \cdot \frac{\partial h^{(k+1)}}{\partial \boldsymbol{x}}=L^{+}\left(h^{(k)}, f_{\mathrm{eq}}\right),
$$

where the derivative with respect to $\boldsymbol{x}$ can be approximated by any conventional CFD schemes such as the finite difference, finite volume, or Discontinuous Galerkin (DG) methods [43, 44], and the collision operator in Eq. (4) can be calculated by the fast spectral method [39, 41] based on the velocity distribution function at the $k$-th iteration step. The process is repeated until relative differences between successive estimates of macroscopic quantities are less than a convergence criterion $\epsilon$.

A key parameter in the rarefied gas dynamics is the rarefaction parameter, which is defined as

$$
\delta_{\mathrm{rp}}=\frac{H}{\lambda}, \lambda=\frac{\mu\left(T_{0}\right) v_{\mathrm{m}}}{n_{0} k_{\mathrm{B}} T_{0}}
$$


where $\mu\left(T_{0}\right)$ is the shear viscosity of the gas at the reference temperature, and $\lambda$ is the mean free path of gas molecules. Alternatively, the Knudsen number is defined as

$$
\mathrm{Kn}=\frac{\sqrt{\pi}}{2 \delta_{\mathrm{rp}}}
$$

The CIS is efficient for highly rarefied gas flow when $\delta_{\mathrm{rp}}$ is very small, where converged solution can be quickly found after several iterations. However, the number of iteration increases significantly with the rarefaction parameter [6, 25]. This is due to the frequent collisions of gas molecules, which quickly smear the perturbance and hinder the fluid information exchange. In order to enhance the information exchange across the whole computational domain, synthetic equations for the evolution of macroscopic flow variables that are asymptotic preserving the Navier-Stokes limit should be developed [6].

To this end, we first multiply Eq. (3) by 1, 2v, and $|\boldsymbol{v}|^{2}-\frac{3}{2}$, respectively, and integrate the resultant equations with respect to $\boldsymbol{v}$; we obtain the following equations for the evolution of density, velocity, and temperature:

$$
\begin{aligned}
\frac{\partial \rho}{\partial t}+\frac{\partial U_{i}}{\partial x_{i}} & =0 \\
2 \frac{\partial U_{i}}{\partial t}+\frac{\partial \rho}{\partial x_{i}}+\frac{\partial T}{\partial x_{i}}+\frac{\partial \sigma_{i j}}{\partial x_{j}} & =0 \\
\frac{3}{2} \frac{\partial T}{\partial t}+\frac{\partial q_{j}}{\partial x_{j}}+\frac{\partial U_{j}}{\partial x_{j}} & =0
\end{aligned}
$$

which are not closed, since expressions for the shear stress $\sigma_{i j}$ and heat flux $\boldsymbol{q}$ are not known. One way to close Eq. (13) is to use the Chapman-Enskog expansion, where the distribution function is expressed in the power series of $\mathrm{Kn}[1]: h=\mathrm{Kn}^{(1)}+\mathrm{Kn}^{2} h^{(2)}+\cdots$. When $f=f^{(0)}$, we have $\sigma_{i j}=q_{i}=0$, and the Euler equations are recovered. When the distribution function is truncated at the first-order of $\mathrm{Kn}$, that is, $h=\mathrm{Kn} h^{(1)}$, we have

$$
\sigma_{i j}=-\delta_{\mathrm{rp}}^{-1}\left(\frac{\partial U_{i}}{\partial x_{j}}+\frac{\partial U_{j}}{\partial x_{i}}-\frac{2}{3} \frac{\partial U_{k}}{\partial x_{k}} \delta_{i j}\right) \equiv-2 \delta_{\mathrm{rp}}^{-1} \frac{\partial U_{<i}}{\partial x_{j}>}, \quad q_{i}=-\frac{5}{4 \operatorname{Pr}} \delta_{\mathrm{rp}}^{-1} \frac{\partial T}{\partial x_{i}},
$$

and Eq. (13) reduces to the Navier-Stokes equations with Pr being the Prandtl number. Higher-order macroscopic equations can be obtained successively but they are not stable. On the other hand, even the obtained high-order macroscopic equations are stable, they are only the approximate solutions of the Boltzmann equation, rather than the exact solutions. Therefore, they cannot describe the multiscale rarefied gas dynamics.

It should be noted that in the implicit UGKS [14] and other variants [45, 46], both the gas kinetic equation and macroscopic equations (13) are solved, where $\sigma_{i j}$ and $\boldsymbol{q}$ are obtained according to Eq. (9). These methods are efficient when the Knudsen number is large, like the CIS. However, in the near-continuum flow regime, the number of iterations are still large, at the order of thousands iterations. The reason for the relative slow convergence is that, if the iteration starts from the global equilibrium state where $\sigma_{i j}$ and $\boldsymbol{q}$ are zero, in most 
of the time the Euler equations, rather than the Navier-Stokes equations that dominate the steady-state flow dynamics, are solved, due to the fact that perturbance from the wall boundary takes a long time to reach the bulk region for near-continuum flows. Even when the shear stress and heat flux are non-zero, solutions of Eq. (13) deviate from that of the Navier-Stokes equations in the near-continuum flow regime unless they nearly converge to the steady-state solution. As a matter of fact, the authors have checked, in the linearized Poiseuille flow [6], that when the kinetic equations is solved according to Eq. (10), Eq. (13) cannot help to find converged solution within dozens of iterations.

Bearing this in mind, to develop an ultra-fast convergence scheme, it is beneficial to construct macroscopic equations that contain the Newton's law for stress and Fourier's law for heat conduction explicitly to recover the macroscopic transport mechanism; that is, the shear stress and heat flux should be expressed as follows:

$$
\begin{aligned}
\sigma_{i j} & =-2 \delta_{\mathrm{rp}}^{-1} \frac{\partial U_{<i}}{\partial x_{j>}}+\operatorname{HoT}_{\sigma_{i j}}, \\
q_{i} & =-\frac{5}{4 \operatorname{Pr}} \delta_{\mathrm{rp}}^{-1} \frac{\partial T}{\partial x_{i}}+\operatorname{HoT}_{q_{i}}
\end{aligned}
$$

where $\mathrm{HoT}_{\sigma_{i j}}$ and $\mathrm{HoT}_{q_{i}}$ are the high-order terms containing contributions of all the orders $O\left(K n^{\alpha^{\prime}}\right)$ with $\alpha^{\prime}=2,3, \cdots, \infty$.

To obtain (15), we multiply Eq. (3) by $2\left(v_{i} v_{j}-\delta_{i j}|\boldsymbol{v}|^{2} / 3\right)$ and integrate the resultant equation with respect to $\boldsymbol{v}$, and obtain

$$
\frac{\partial \sigma_{i j}}{\partial t}+\underbrace{2 \int\left(v_{i} v_{j}-\frac{\delta_{i j}}{3}|\boldsymbol{v}|^{2}\right) \boldsymbol{v} \cdot \frac{\partial h}{\partial \boldsymbol{x}} \mathrm{d} \boldsymbol{v}-2 \frac{\partial U_{<i}}{\partial x_{j>}}}_{\text {HoT }}+\underbrace{2 \frac{\partial U_{<i}}{\partial x_{j>}}=-\delta_{\mathrm{rp}} \sigma_{i j}}_{\text {Newton's law of viscosity }}+2 \int\left(L-L_{\mathrm{s}}\right) v_{i} v_{j} \mathrm{~d} \boldsymbol{v}
$$

where

$$
L_{\mathrm{s}}=\delta_{\mathrm{rp}}\left\{\left[\rho+2 \boldsymbol{U} \cdot \boldsymbol{v}+T\left(|\boldsymbol{v}|^{2}-\frac{3}{2}\right)+\frac{4(1-\mathrm{Pr})}{5} \boldsymbol{q} \cdot \boldsymbol{v}\left(|\boldsymbol{v}|^{2}-\frac{5}{2}\right)\right] f_{\mathrm{eq}}-h\right\}
$$

is the linearized collision operator of the Shakhov kinetic model equation [47], and

$$
\operatorname{HoT}_{\sigma_{i j}}=\left\{\begin{array}{r}
\frac{\partial}{\partial x_{i}} \int\left(2 v_{i}^{2}-1\right) v_{j} h \mathrm{~d} \boldsymbol{v}+\frac{\partial}{\partial x_{j}} \int\left(2 v_{j}^{2}-1\right) v_{i} h \mathrm{~d} \boldsymbol{v}+\frac{\partial}{\partial x_{k}} \int 2 v_{1} v_{2} v_{3} h \mathrm{~d} \boldsymbol{v}, \\
\text { for } i \neq j, k \neq i, k \neq j, \\
\frac{\partial}{\partial x_{i}} \int 2\left(v_{i}^{2}-\frac{|\boldsymbol{v}|^{2}}{3}-\frac{2}{3}\right) v_{i} h \mathrm{~d} \boldsymbol{v}+\sum_{k} \frac{\partial}{\partial x_{k}} \int 2\left(v_{i}^{2}-\frac{|\boldsymbol{v}|^{2}}{3}+\frac{1}{3}\right) v_{k} h \mathrm{~d} \boldsymbol{v}, \\
\text { for } i=j, k \neq i .
\end{array}\right.
$$

Note that this derivation is simple as we just separate the underlined term in Eq. (17) from high-order moments $\int 2\left(v_{i} v_{j}-\delta_{i j}|\boldsymbol{v}|^{2} / 3\right) v_{k} h \mathrm{~d} \boldsymbol{v}$, and the purpose of introducing $L_{\mathrm{s}}$ is only to recover the term $\delta_{\mathrm{rp}} \sigma_{i j}$, so that the Newton's law of stress is recovered explicitly. It should also be noted that, for the linearized Boltzmann collision operator, the term $2 \int\left(L-L_{\mathrm{s}}\right) v_{i} v_{j} \mathrm{~d} \boldsymbol{v}$ is negligible when compared to $\delta_{\mathrm{rp}} \sigma_{i j}$. For instances, for the Maxwell 
molecular model, this term is zero, while for the HS molecular model, this term is less than $2 \%$ of $\delta_{\mathrm{rp}} \sigma_{i j}$, see page no. 169 in the third edition of the book [1].

Similarly, to obtain Eq. (16), we multiply Eq. (3) by $v_{i}\left(|\boldsymbol{v}|^{2}-5 / 2\right)$ and integrate the resultant equation with respect to $\boldsymbol{v}$; we obtain

$$
\frac{\partial q_{i}}{\partial t}+\mathrm{HoT}_{q_{i}}+\frac{3 C_{q}}{2} \frac{\partial T}{\partial x_{i}}=-\frac{2}{3} \delta_{\mathrm{rp}} q_{i}+\int\left(L-L_{\mathrm{s}}\right) v_{i}|\boldsymbol{v}|^{2} \mathrm{~d} \boldsymbol{v}
$$

where

$$
\operatorname{HoT}_{q_{i}}=\frac{\partial}{\partial x_{i}} \int\left[\left(v_{i}^{2}-C_{q}\right)\left(|\boldsymbol{v}|^{2}-\frac{3}{2}\right)-v_{i}^{2}\right] h \mathrm{~d} \boldsymbol{v}+\sum_{j \neq i} \frac{\partial}{\partial x_{j}} \int v_{i} v_{j}\left(|\boldsymbol{v}|^{2}-\frac{5}{2}\right) h \mathrm{~d} \boldsymbol{v} .
$$

For the linearized Boltzmann collision operator, the term $\int\left(L-L_{\mathrm{s}}\right) v_{i}|\boldsymbol{v}|^{2} d \boldsymbol{v}$ is negligible when compared to $\delta_{\mathrm{rp}} q_{i}$, i.e. within $3 \%$ of $\delta_{\mathrm{rp}} q_{i}$ [1]. If we choose $C_{q}=5 / 9 \mathrm{Pr}$, then the under-braced term in Eq. (20) recovers the Fourier's heat conduction law in Eq. (14). Since for monatomic gas the Prandtl number is very close to $2 / 3$, in the following paper we choose $C_{q}=5 / 6$.

Note that the macroscopic equations (13), (17) and (20) resemble the Grad 13 moment equations [48, 49]. However, since the higher-order terms (19) and (21) are computed directly from the velocity distribution function, no approximations are introduced here. If the velocity distribution function is approximated by the Gauss-Hermite polynomials to the third order, where the coefficients before those polynomials are determined by the first 13 moments of the velocity distribution function, then G13 moment equations will be recovered. Since the first-order Chapman-Enskog expansion to G13 equations leads to Eqs. (13) and (14), that is, only the underlined terms in Eqs. (17) and (20) are retained, the derived synthetic equations (13), (17) and (20) are asymptotic preserving the Navier-Stokes limit. Thus, they should be able to boost the convergence to the steady-state solution of the LBE significantly, as in the bulk region (a few mean free path of gas molecules away from solid surfaces) we are effectively solving the Navier-Stokes equations.

With these macroscopic equations to update the macroscopic quantities and the velocity distribution function, we devise the following iteration scheme to find the steady-state solution of the LBE (3) efficiently:

- Step 1. When the velocity distribution function $h^{(k)}$ and the corresponding macroscopic quantities in Eqs. (8) and (9) are known at the $k$-th iteration, we calculate $2 \int\left(L-L_{\mathrm{s}}\right) v_{i} v_{j} \mathrm{~d} \boldsymbol{v}$ in Eq. (17) and $\int\left(L-L_{\mathrm{s}}\right) v_{i}|\boldsymbol{v}|^{2} \mathrm{~d} \boldsymbol{v}$ in Eq. (20). We also calculate the velocity distribution function $h^{(k+1 / 2)}$ according to the conventional iterative scheme (10), that is, we solve the following equation:

$$
\nu_{\mathrm{eq}}(\boldsymbol{v}) h^{(k+1 / 2)}+\boldsymbol{v} \cdot \frac{\partial h^{(k+1 / 2)}}{\partial \boldsymbol{x}}=L^{+}\left(h^{(k)}, f_{\mathrm{eq}}\right),
$$

by a second-order upwind finite difference in the bulk and a first-order upwind scheme at the solid surface [42] or the DG method [43, 44]. 
- Step 2. From $h^{(k+1 / 2)}$, we calculate the density $\rho^{(k+1 / 2)}(\boldsymbol{x})$, flow velocity $\boldsymbol{U}^{(k+1 / 2)}(\boldsymbol{x})$, the temperature $T^{(k+1 / 2)}(\boldsymbol{x})$, the shear stress $\sigma_{i j}^{(k+1 / 2)}(\boldsymbol{x})$, the heat flux $\boldsymbol{q}^{(k+1 / 2)}(\boldsymbol{x})$, and the high-order terms $\mathrm{HoT}_{\sigma_{i j}}$ and $\mathrm{HoT}_{q_{i}}$ defined in Eqs. 19) and (21), respectively.

- Step 3. We obtain the macroscopic quantities $\rho^{(k+1)}(\boldsymbol{x}), \boldsymbol{U}^{(k+1)}(\boldsymbol{x}), T^{(k+1)}(\boldsymbol{x}), \sigma_{i j}^{(k+1)}(\boldsymbol{x})$, and $\boldsymbol{q}^{(k+1)}(\boldsymbol{x})$ by solving the synthetic equations (13), (17) and (20), That is, for the steady-state problems the shear stress and heat flux can be solved from Eq. (17) and (20), which will then be substituted to Eq. (13) to form the Navier-Stokes equations with source terms related to the higher-order terms defined in Eqs. (19) and (21). These equation can be solved by the SIMPLE algorithm and/or DG method easily in the bulk region, where the boundary values in the vicinity of wall for the density, velocity, temperature are obtained from Step 2. The detailed DG algorithm to solve the synthetic equations can be found in the Appendix.

- Step 4. The velocity distribution function $h$ is modified to incorporate the change of macroscopic quantities. That is,

$$
\begin{aligned}
h^{(k+1)}(\boldsymbol{x}, \boldsymbol{v})= & h^{(k+1 / 2)}(\boldsymbol{x}, \boldsymbol{v})+\left[2 \lambda_{\boldsymbol{U}}(\boldsymbol{x}) \cdot \boldsymbol{v}+\frac{4}{5} \lambda_{\boldsymbol{q}}(\boldsymbol{x}) \cdot \boldsymbol{v}\left(|\boldsymbol{v}|^{2}-\frac{5}{2}\right)\right] f_{e q} \\
& +\left[\lambda_{\rho}(\boldsymbol{x})+\lambda_{T}(\boldsymbol{x})\left(|\boldsymbol{v}|^{2}-\frac{3}{2}\right)+\lambda_{\sigma_{i j}}(\boldsymbol{x})\left(v_{i} v_{j}-\frac{|\boldsymbol{v}|^{2}}{3} \delta_{i j}\right)\right] f_{e q}
\end{aligned}
$$

where $\lambda_{\boldsymbol{U}}(\boldsymbol{x})=\boldsymbol{U}^{(k+1)}(\boldsymbol{x})-\boldsymbol{U}^{(k+1 / 2)}(\boldsymbol{x}), \lambda_{\boldsymbol{q}}(\boldsymbol{x})=\boldsymbol{q}^{(k+1)}(\boldsymbol{x})-\boldsymbol{q}^{(k+1 / 2)}(\boldsymbol{x}), \lambda_{\rho}(\boldsymbol{x})=$ $\rho^{(k+1)}(\boldsymbol{x})-\rho^{(k+1 / 2)}(\boldsymbol{x}), \lambda_{T}(\boldsymbol{x})=T^{(k+1)}(\boldsymbol{x})-T^{(k+1 / 2)}(\boldsymbol{x})$, and $\lambda_{\sigma_{i j}}(\boldsymbol{x})=\beta \sigma_{i j}^{(k+1)}(\boldsymbol{x})-$ $\beta \sigma_{i j}^{(k+1 / 2)}(\boldsymbol{x})$, with $\beta=3 / 2$ when $i=j$ and $\beta=2$ otherwise.

- Step 5. The above steps are repeated until convergence.

Since the gas kinetic equation is solved together with the macroscopic equations (13), (17) and (20) for general rarefied gas flows, the above scheme is called the GSIS. Note that although the SIS has been widely applied to the radiation transport process [24] and rarefied gas flows driven by local pressure, temperature, and concentration gradients [25, 30, 31, 27, 43. to overcome the slow convergence and remove the constraint on the spatial cell size in the near-continuum flow regime, it is the first time that the GSIS is developed for general rarefied gas flows described by the LBE. Also, it is with no doubt that such a methodology can be directly applied to construct the GSIS for the nonlinear Boltzmann equation.

\section{Numerical test for zero-dimensional problem: Rayleigh-Brillouin scattering}

For linearized problems, if the external force that drives the flow changes periodically in time, then the velocity distribution function can be expressed as [50, 51, 52]:

$$
f=f_{\mathrm{eq}}(\boldsymbol{v})+\alpha \Re[\exp (i \mathrm{St} t) h(\boldsymbol{x}, \boldsymbol{v})]
$$


where $\Re$ is the real part of a variable and $h$ satisfies the following linearized Boltzmann equation:

$$
i \mathrm{St} h+\boldsymbol{v} \cdot \frac{\partial h}{\partial \boldsymbol{x}}=L\left(h, f_{\mathrm{eq}}\right) .
$$

Note that here $i$ is the imaginary unit and $h$ is a complex function, so are the macroscopic quantities defined in Eqs. (8) and (9). These complex values will introduce phase shifts relative to that of the external driving force. The Strouhal number St

$$
\mathrm{St}=\frac{\varpi H}{v_{\mathrm{m}}}
$$

is the oscillation frequency $\varpi$ normalized by $v_{\mathrm{m}} / H$. The solutions to these oscillating problems can also be accelerated by the GSIS; the corresponding macroscopic synthetic equations can be obtained if we replace $\partial / \partial t$ in Sec. 3 by $i$ St.

In the spontaneous Rayleigh-Brillouin scattering (SRBS), light propagating through the gas is scattered by the thermal motion of gas molecules, where the spectrum of the scattered light contains the information of gas such as temperature, speed, and viscosity. Thus, SRBS provides a non-intrusive way to probe the gas properties in a remote way. Theoretically, the SRBS spectrum can be obtained by solving the LBE (3) with the initial condition $h\left(t=0, x_{2}, \boldsymbol{v}\right) \propto \delta\left(x_{2}\right) f_{\text {eq }}(\boldsymbol{v})$, which represents a density impulse [53, 54]. To be more specific, the SRBS spectrum is calculated as

$$
S_{s}\left(\delta_{\mathrm{rp}}, f_{s}\right)=\Re\left(\int \hat{h} \mathrm{~d} \boldsymbol{v}\right)
$$

where $\hat{h}(\boldsymbol{v})$, the Laplace-Fourier transforms of $h$ in the temporal and spatial directions, satisfies (suppose the scattered light propagates in the $x_{2}$ direction)

$$
2 \pi i\left(f_{s}-v_{2}\right) \hat{h}=L^{+}(\hat{h})-\nu_{\text {eq }} \hat{h}+f_{\text {eq }} .
$$

Note that in Eq. (27) and (28), the rarefaction parameter $\delta_{\mathrm{rp}}$ is defined when the characteristic flow length $H$ is $\lambda_{L} / 2 \sin \left(\theta_{s} / 2\right)$, with $\lambda_{L}$ being the wavelength of laser and $\theta_{s}$ the angle of light scattering, and $f_{s}(=\mathrm{St} / 2 \pi)$ is the frequency shift in the scattering process normalized by the characteristic frequency $v_{m} / H$. Also note that terms in the left-handside of Eq. (28) appear because operators $\partial / \partial t$ and $\partial / \partial x_{2}$ in Eq. (3) are replaced by $2 i \pi f_{s}$ and $-2 i \pi$, respectively. Finally, the source term $f_{\text {eq }}$ in Eq. (28) is from the Laplace transform of the initial density impulse. This term will change the first equation in Eq. (13) to $\frac{\partial \rho}{\partial t}+\frac{\partial U_{i}}{\partial x_{i}}=1$, while other synthetic equations remain unchanged.

In CIS, the velocity distribution function is obtained by solving the following equation iteratively:

$$
\hat{h}^{(k+1)}(\boldsymbol{v})=\frac{L^{+}\left(\hat{h}^{(k)}\right)+f_{\mathrm{eq}}(\boldsymbol{v})}{2 \pi i\left(f_{s}-v_{2}\right)+\nu_{\mathrm{eq}}(\boldsymbol{v})},
$$

which converges fast when $\delta_{\mathrm{rp}}$ is small, but extremely slow when $\delta_{\mathrm{rp}}$ is large as the flow enters the near-continuum regime. 

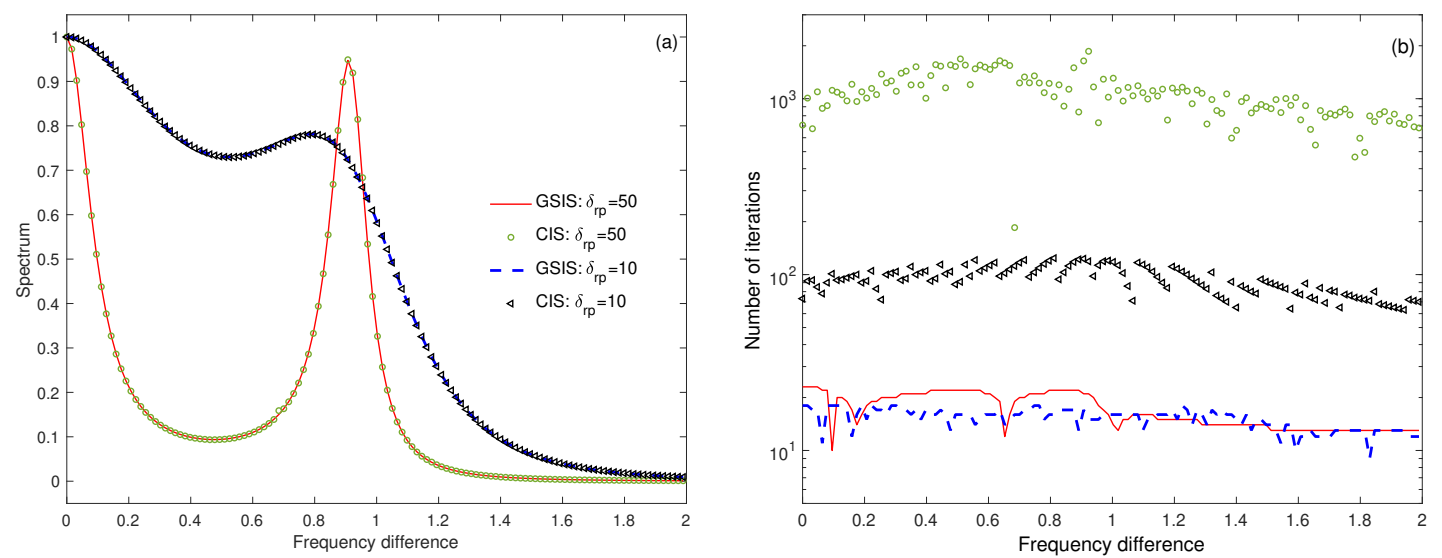

Figure 1: Comparisons of the SRBS spectrum (a) and iteration numbers (b) between the CIS and GSIS when the rarefaction parameter is large. The HS molecular model is used in the LBE. The molecular velocity space $[-6,6]^{3}$ is discretized by $24 \times 32 \times 24$ uniformly distributed points. The solutions are believed to be converged when the relative error in $\hat{\rho}$ between two consecutive iteration steps is less than $10^{-7}$.

In the GSIS, the synthetic equations can be rewritten in the following matrix at the $(k+1)$-th iteration step:

$$
\left[\begin{array}{ccccc}
2 i \pi f_{s} & -2 i \pi & 0 & 0 & 0 \\
-2 i \pi & 4 i \pi f_{s} & -2 i \pi & -2 i \pi & 0 \\
0 & -2 i \pi & 3 i \pi f_{s} & 0 & -2 i \pi \\
0 & -\frac{8}{3} i \pi & 0 & 2 i \pi f_{s}+\delta_{r p} & 0 \\
0 & 0 & -3 i \pi C_{q} & 0 & 2 i \pi f_{s}+\frac{2}{3} \delta_{r p}
\end{array}\right]\left[\begin{array}{c}
\hat{n}^{(k+1)} \\
\hat{U}_{2}^{(k+1)} \\
\hat{T}^{(k+1)} \\
\hat{\sigma}_{22}^{(k+1)} \\
\hat{q}_{2}^{(k+1)}
\end{array}\right]=\left[\begin{array}{c}
1 \\
0 \\
0 \\
\mathrm{R}_{4} \\
\mathrm{R}_{5}
\end{array}\right],
$$

where, the hat denotes the Laplace-Fourier transform of the corresponding quantity, $\mathrm{R}_{4}=$ $2 i \pi \mathrm{HoT}_{\sigma_{22}}^{(k+1 / 2)}+2 \int\left(L-L_{\mathrm{s}}\right) v_{2}^{2} \mathrm{~d} \boldsymbol{v}$ and $\mathrm{R}_{5}=2 i \pi \mathrm{HoT}_{q_{2}}^{(k+1 / 2)}+\int\left(L-L_{\mathrm{s}}\right) v_{2}|\boldsymbol{v}|^{2} \mathrm{~d} \boldsymbol{v}$.

In the numerical simulation, starting from the zero perturbance at each frequency difference, solutions are believed to be converged when the relative error in $\hat{\rho}$ between two consecutive iteration steps is less than $10^{-7}$. Results in Fig. 1(a) show that GSIS and CIS generate almost the same SRBS spectra, except at $\delta_{\mathrm{rp}}=50$ the CIS has a false converged solution (i.e. the discontinuous spectrum) when the frequency difference is around 0.68. As usual, the iteration number in CIS increases significantly with the rarefaction parameter $\delta_{\mathrm{rp}}$, while in GSIS this remains nearly unchanged and is far less than that of the CIS. For example, the iteration number of the GSIS is about 10 and 100 times less than that of the CIS when $\delta_{\mathrm{rp}}=10$ and 50, respectively. We have also tested that, even when $\delta_{\mathrm{rp}}=500$, converged solutions are obtained within 20 steps in the GSIS for every frequency difference.

However, when $\delta_{\text {rp }}$ is small and St is large, the GSIS does not converge or even blows up. This is because the eigenvalue of the matrix in Eq. (30) has large complex values so that any inappropriate initial guess can lead to large oscillations that decay rather slow or even blow up. Whereas, physically speaking, the solution should decay fast due to the large rarefaction 


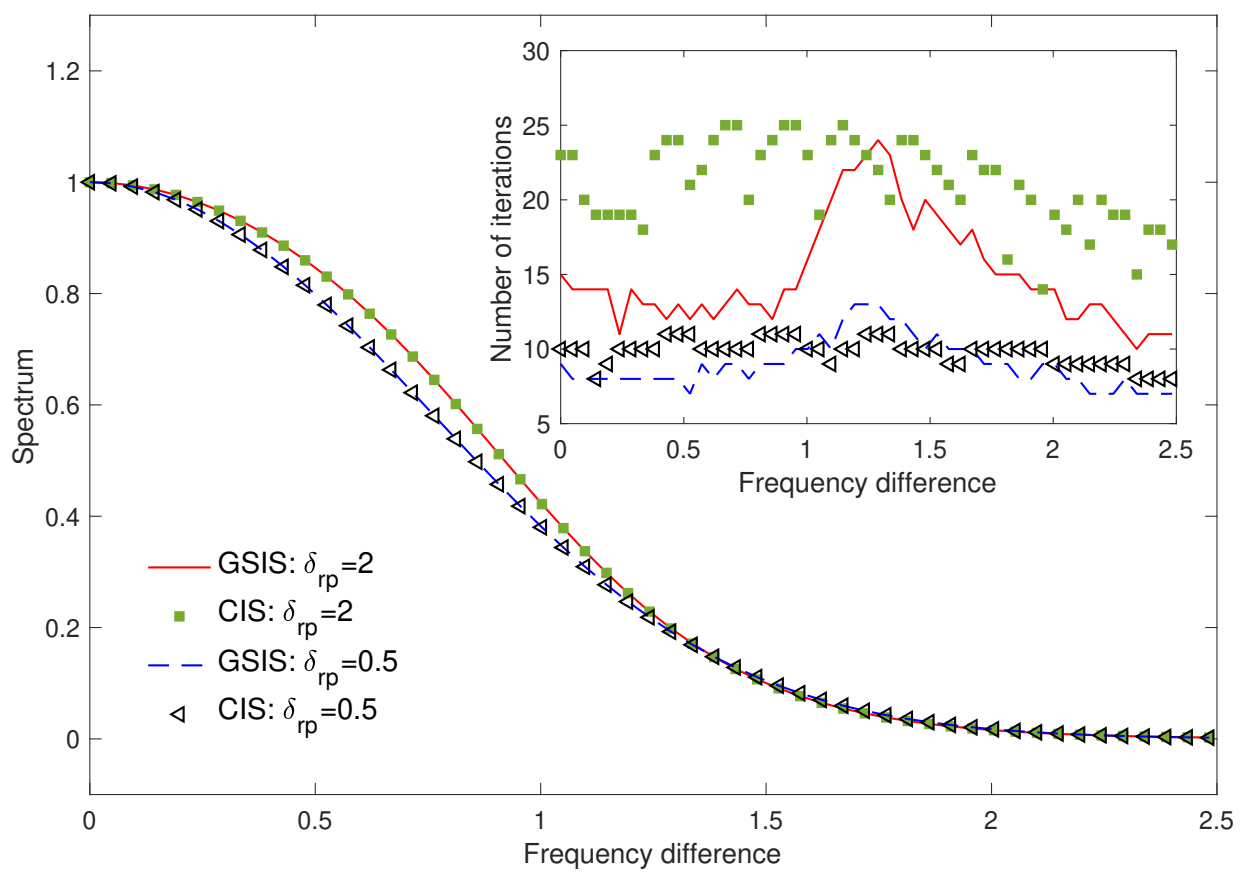

Figure 2: Comparisons of the SRBS spectrum and iteration numbers (inset) between the CIS and GSIS when the rarefaction parameter is small. The Maxwell molecular model is used in the LBE. The molecular velocity space $[-6,6]^{3}$ is discretized by $24 \times 192 \times 24$ uniformly distributed points due to high rarefaction effects. The solutions are believed to be converged when the relative error in $\hat{\rho}$ between two consecutive iteration steps is less than $10^{-7}$.

effect, that is, large dissipation. To remedy this, the small value $\delta_{\mathrm{rp}}$ in the left-hand side of Eq. (30) is replaced by a relative large value $\bar{\delta}_{\mathrm{rp}}=\max \left(\delta_{\mathrm{rp}}, 10\right)$, while the right-hand side terms are modified correspondingly as

$$
\begin{aligned}
& \mathrm{R}_{4}=2 i \pi \operatorname{HoT}_{\sigma_{22}}^{(k+1 / 2)}+2 \int\left(L-L_{\mathrm{s}}\right) v_{2}^{2} \mathrm{~d} \boldsymbol{v}+\left(\bar{\delta}_{\mathrm{rp}}-\delta_{\mathrm{rp}}\right) \hat{\sigma}_{22}^{(k+1 / 2)} \\
& \mathrm{R}_{5}=2 i \pi \operatorname{HoT}_{q_{2}}^{(k+1 / 2)}+\int\left(L-L_{\mathrm{s}}\right) v_{2}|\boldsymbol{v}|^{2} \mathrm{~d} \boldsymbol{v}+\frac{2}{3}\left(\bar{\delta}_{\mathrm{rp}}-\delta_{\mathrm{rp}}\right) \hat{q}_{2}^{(k+1 / 2)} .
\end{aligned}
$$

This simple treatment helps to decay non-physical solutions at initial few iteration steps. When the solution of the new system converges, it can be proven that it satisfies Eq. (30). Therefore, no approximation is introduced to the converged solution. This point is proven in Fig. 2, where the GSIS and CIS solutions agree perfectly with each other, and from the inset we see that the GSIS needs slightly less iteration steps than CIS in most of frequency differences.

Another remarkable property of the GSIS is that, at the same level of convergence criterion, the GSIS provides more accurate numerical solutions. One example is given in Fig. 3, where one can see that the relative error between two consecutive iteration steps $\epsilon=\left|\hat{\rho}^{(k+1)} / \hat{\rho}^{(k)}-1\right|$ decays rather fast in the GSIS, while in the CIS it decreases slowly with 

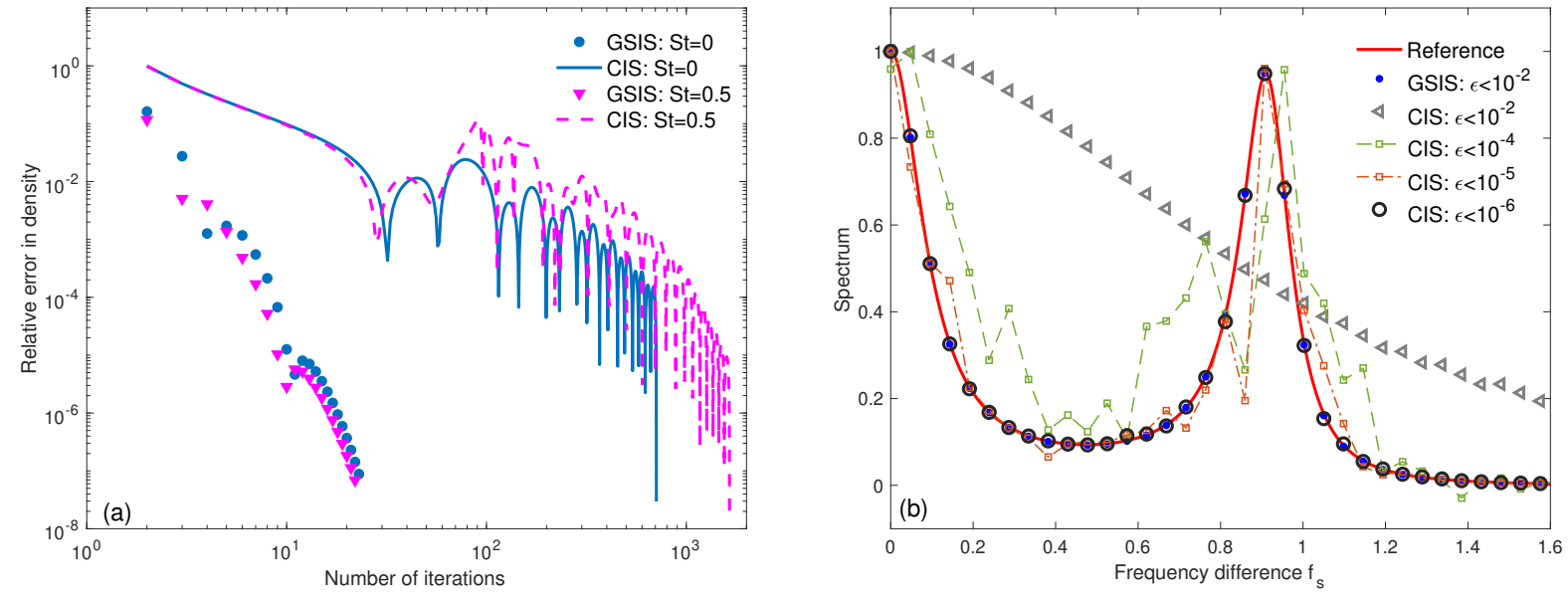

Figure 3: (a) The decay of the relative error $\epsilon=\left|\hat{\rho}^{(k+1)} / \hat{\rho}^{(k)}-1\right|$ between two consecutive iteration steps, and (b) the SRBS spectra obtained at different level of convergence criterion. The reference solution is obtained from the GSIS when $\epsilon=10^{-7}$. The linearized Boltzmann equation with HS molecular model is used, with the rarefaction parameter $\delta_{\mathrm{rp}}=50$.

many oscillations. As a consequence, the GSIS finds the correct spectrum profile even when the relative error in density is less $10^{-2}$, while the CIS can only reach the correct solution when the error is less than $10^{-6}$. This is explained below. According to the analysis of Adam and Larsen for radiation transfer problem [24], if one stops at the $(k+1)$-th step with

$$
\left|\frac{\hat{\rho}^{(k+1)}}{\hat{\rho}^{(k)}}-1\right|=\epsilon
$$

in the CIS, then the relative difference from the true solution $\hat{\rho}$ is

$$
\left|\frac{\hat{\rho}}{\hat{\rho}^{(k+1)}}-1\right| \approx \frac{\gamma}{1-\gamma} \epsilon
$$

where $\gamma$ is the spectral radius of the iteration operator. For problem with slow convergence, $\gamma$ is very close to one (see Figure 1 in Ref. [6] for the kinetic BGK model equation), which could make the difference from true solution magnified by thousands of times. And thus the convergence criterion $\epsilon$ in CIS should be set at a much smaller value.

\section{Numerical tests for one-dimensional problems}

Numerical simulations are further carried out to assess efficiency and accuracy of the GSIS. To this end, we consider one-dimensional problems between two parallel plates, including the Fourier flow, oscillating Couette flow and sound prorogation. The reason is that in previous cases the special SIS is only applicable for rarefied gas flows [25, 30, 31, 27, 43, 33], where the flow velocity is perpendicular to the computational domain. Here we investigate the performance of GSIS for typical general rarefied gas flows, where the flow velocity (or other macroscopic variables) also varies within the computational domain. 


\subsection{Heat transfer between two parallel plates}

Consider the steady Fourier flow of a gas between two infinite parallel plates with a distance $H$, located at $x_{2}=0$ and $x_{2}=1$. The two plates are stationary, the one at $x_{2}=0$ has a temperature $T_{0}-\Delta T / 2$, while that at $x_{2}=H$ has a temperature $T_{0}+\Delta T / 2$. We assume that the temperature difference $\Delta T$ is negligible compared to $T_{0}$, so that the problem is symmetrical around $x_{2}=1 / 2$. Therefore, in numerical simulations only the region $x_{2} \in[0,1 / 2]$ is considered. The Boltzmann equation is linearized by choosing $\alpha=\Delta T / T_{0}$ in Eq. (2). The boundary condition at $x_{2}=0$, as according to Eqs. (2) and (7), is

$$
h\left(x_{2}=0, \boldsymbol{v}\right)=\left[1-\frac{|\boldsymbol{v}|^{2}}{2}-2 \sqrt{\pi} \int_{v_{2}<0} v_{2} h\left(x_{2}=0, \boldsymbol{v}\right) \mathrm{d} v_{2}\right] f_{\text {eq }}, \quad \text { when } v_{2}>0,
$$

while that at $x_{2}=0.5$ is

$$
h\left(v_{1}, v_{2}, v_{3}\right)=-h\left(v_{1},-v_{2}, v_{3}\right),
$$

due to the symmetry of this linearized problem.

From the synthetic equations (13), (17) and (20), as well as the symmetry condition (35), we know

$$
\boldsymbol{U}=0, \quad \sigma_{i j}=0 \quad \text { when } i \neq j, \quad q_{1}=q_{3}=0,
$$

the heat flux perpendicular to the two plates $q_{2}$ is a constant, and the variation of the perturbed temperature satisfies

$$
\frac{\partial T}{\partial x_{2}}=-\frac{4 \delta_{\mathrm{rp}}}{9 C_{q}} q_{2}+\underbrace{\frac{2}{3 C_{q}} \int v_{2}|\boldsymbol{v}|^{2}\left(L-L_{\mathrm{s}}\right) \mathrm{d} \boldsymbol{v}}_{H_{1}^{(k)}\left(x_{2}\right)}-\underbrace{\frac{2}{3 C_{q}} \frac{\partial}{\partial x_{2}} \int\left(v_{2}^{2}-C_{q}\right)\left(|\boldsymbol{v}|^{2}-\frac{3}{2}\right) h \mathrm{~d} \boldsymbol{v}}_{H_{2}^{(k+1 / 2)}\left(x_{2}\right)}
$$

whose solution at the $(k+1)$-th iteration step is given by

$$
T^{(k+1)}\left(x_{2}\right)=-\frac{4 \delta_{\mathrm{rp}} q_{2}}{9 C_{q}}\left(x_{2}-\frac{1}{2}\right)+\int_{1 / 2}^{x_{2}} H_{1}^{(k)}\left(x_{2}\right) \mathrm{d} x_{2}-H_{2}^{(k+1 / 2)}\left(x_{2}\right),
$$

where the constant heat flux $q_{2}$ is

$$
q_{2}=\frac{9 C_{q}}{2 \delta_{\mathrm{rp}}}\left[T^{(k+1 / 2)}\left(x_{2}=0\right)+H_{2}^{(k+1 / 2)}\left(x_{2}=0\right)-H_{1}^{(k)}\left(x_{2}=0\right)\right] .
$$

When the temperature is known, the density variation can be easily obtained by solving the following equation

$$
\rho+T+\sigma_{22}=\int 2 v_{2}^{2} h \mathrm{~d} \boldsymbol{v},
$$

where the term at the right-hand-side of Eq. (40) is zero due to the symmetry condition (35), and according to Eq. (17) the stress $\sigma_{22}$ can be calculated as

$$
\sigma_{22}=-\frac{\frac{\partial}{\partial x_{2}} \int 2\left(v_{2}^{2}-\frac{|\boldsymbol{v}|^{2}}{3}\right) v_{2} h \mathrm{~d} \boldsymbol{v}}{\delta_{\mathrm{rp}}}+\frac{2}{\delta_{\mathrm{rp}}} \int\left(L-L_{\mathrm{s}}\right) v_{2}^{2} \mathrm{~d} \boldsymbol{v}
$$



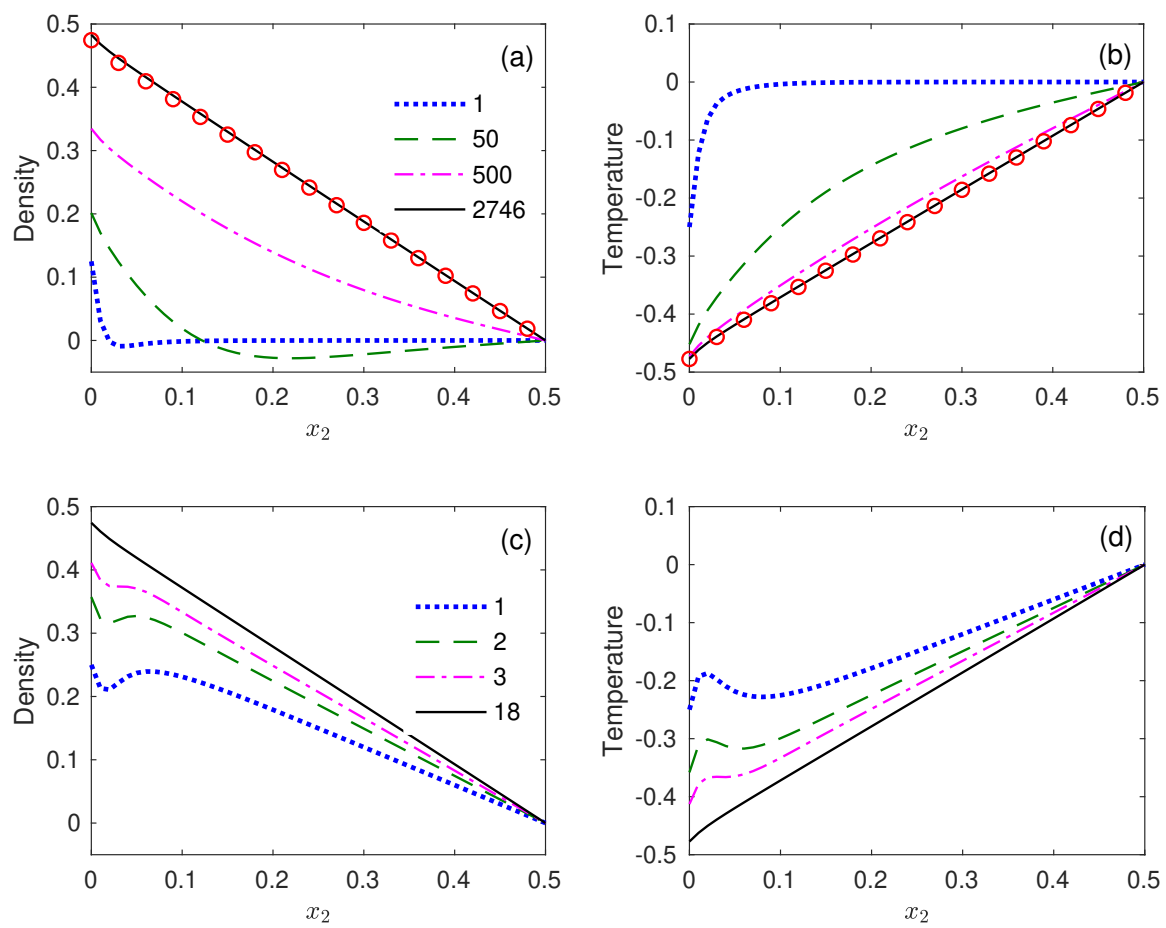

Figure 4: Density and temperature profiles at different iteration steps obtained from the CIS (a, b) and GSIS $(\mathrm{c}, \mathrm{d})$, when the rarefaction parameter is $\delta_{\mathrm{rp}}=50$. Circles show the converged solution obtained from the GSIS. The linearized Shakhov model is used with the initial condition $h\left(x_{2}, \boldsymbol{v}\right)=0$. The spatial region is discretized by $N_{2}=51$ equidistant points. The iteration stops when $\epsilon$ in Eq. (43) is less than $10^{-5}$. Data in the legends are the iteration steps.

We first test efficiency of the GSIS based on the Shakhov model, that is, the linearized Boltzmann collision operator in Eq. (3) is replaced by the linearized Shakhov model (18). We choose the rarefaction parameter $\delta_{\mathrm{rp}}=50$ and discretize the half spatial space into $N_{2}$ even-spaced points, where the derivative with respect to $x_{2}$ is approximated by a secondorder upwind finite difference. The molecular velocity space in the $v_{1}$ and $v_{3}$ directions is truncated to the region $[-6,6]$ by $24 \times 24$ equidistant points, while the molecular velocity $v_{2}$ is truncated to $[-6,6]$ and approximated by the non-uniform points [39, 55]:

$$
v_{2}=\frac{6}{\left(N_{\mathrm{v}}-1\right)^{\imath}}\left[\left(-N_{\mathrm{v}}+1\right)^{\imath},\left(-N_{\mathrm{v}}+3\right)^{\imath}, \cdots,\left(N_{\mathrm{v}}-1\right)^{\imath}\right]
$$

which is useful to capture the discontinuity in the velocity distribution function near $v_{2} \sim 0$. In this test we take $\imath=3$ and $N_{\mathrm{v}}=64$. The iterations in both CIS and GSIS are terminated when

$$
\epsilon=\max \left\{\int\left|\frac{\rho^{(k+1)}}{\rho^{(k)}}-1\right| \mathrm{d} x_{2}, \int\left|\frac{T^{(k+1)}}{T^{(k)}}-1\right| \mathrm{d} x_{2}, \int\left|\frac{q_{2}^{(k+1)}}{q_{2}^{(k)}}-1\right| \mathrm{d} x_{2}\right\}
$$

is less than a certain value. Note that $\rho$ and $T$ at $x_{2}=1 / 2$ are excluded in the above equation since they are zero. 


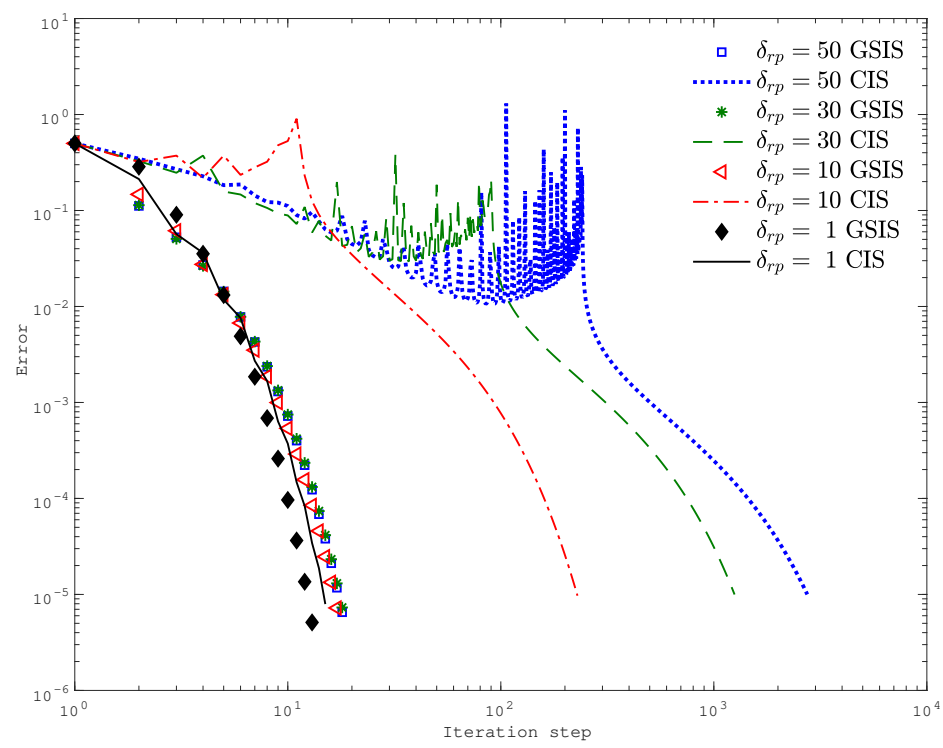

Figure 5: The decay of the error $\epsilon$ as a function of the iteration step, for the Fourier flow between two parallel plates described by the linearized Shakhov model. The spatial region is discretized by $N_{2}=51$ equidistant points.

Figure 4 compares the convergence history of the GSIS and CIS when the rarefaction parameter is $\delta_{\mathrm{rp}}=50$, that is, the flow is in the near-continuum regime. Starting from the initial guess $h\left(x_{2}, \boldsymbol{v}\right)=0$, the perturbance from the solid surface quickly changes the density and temperature near the solid surface in the CIS (within about one molecular mean free path away from the wall). However, due to the frequent collision between gas molecules, it takes a long time (i.e. iteration steps) to feel this change in the bulk region. From example, from Fig. 4(b) we see that about 50 iteration steps are taken for the temperature at $x_{2}=0.5$ to feel this change. Moreover, such a change does not necessarily lead to the final converged state monotonically, but it could deviate further away from the final steady state: from Fig. 4(a) we see that the density perturbance in the bulk region is even negative after 50 iterations, while the final steady state of the density is always non-negative in the region of $x_{2} \in[0,0.5]$. This is also evidenced in Fig. 5 that the error does not decay monotonically but oscillates several times. Such a slow convergence is completely changed in the GSIS, where the temperature and density are corrected according to the synthetic equations (37) and 40); the dominated parts are respectively $\frac{\partial T}{\partial x_{2}}=-\frac{4 \delta_{\mathrm{rp}}}{9 C_{q}} q_{2}$ and $\rho=-T$ when $\delta_{\mathrm{rp}}$ is large, and this means that the temperature and density in the bulk region are corrected to be nearly linear immediately. As we can see from Fig. 4(d), after the first iteration, the temperature from the GSIS at $x_{2}=0$ is the same as that from the CIS, but the temperature from the GSIS in the bulk region varies linearly, while that from the CIS is still zero. From Fig. 4(c) we see that the density also varies linearly in the bulk, but at the solid surface it is more close to the final state than that obtained from the CIS. Since the diffusion-type macroscopic equation (37) allows the efficient exchange of information, fast convergence is realized in the whole computational domain, see Fig. 4(c) and (d). 
(a)

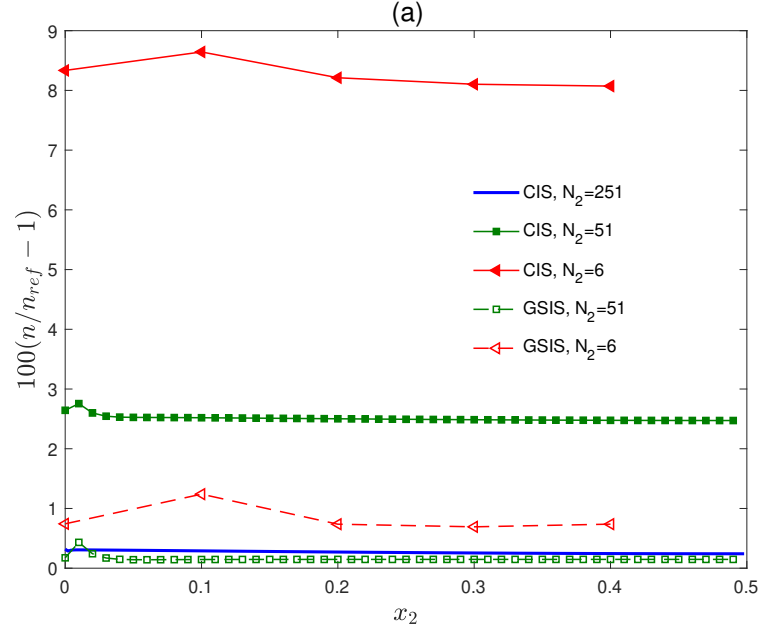

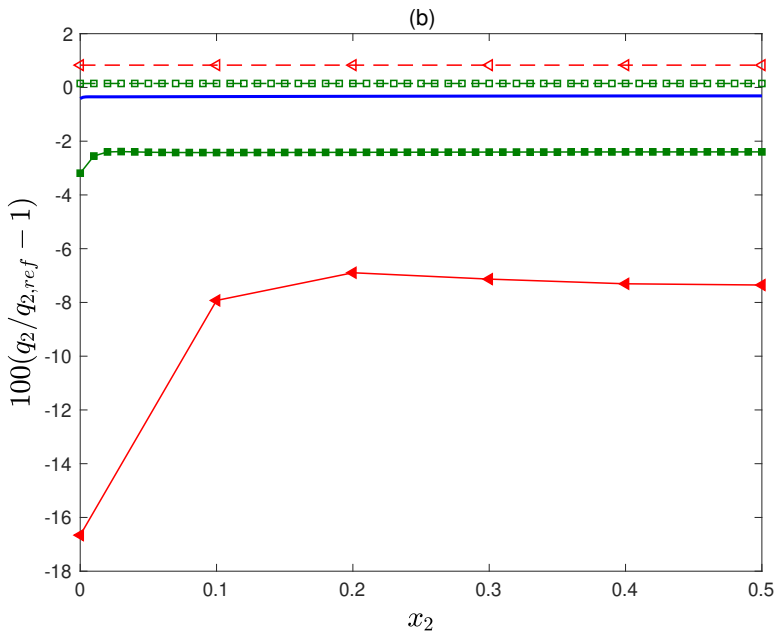

Figure 6: The influence of the spatial discretization on accuracy of both the CIS and GSIS, for the Fourier flow between two parallel plates described by the linearized Shakhov model with $\delta_{\mathrm{rp}}=50$. The iteration terminates when $\epsilon<10^{-6}$. The reference solutions (i.e. $\rho_{\text {ref }}$ and $q_{2 \text {,ref }}$ ) are obtained from the GSIS with $N_{2}=251$, that is, the spatial cell size is about one tenth of the mean free path of gas molecules.

Figure 5 demonstrates how fast the solution is converged at different values of rarefaction parameter. When $\delta_{\mathrm{rp}}$ is small, errors in both the CIS and GSIS decay at the same rate, which means that the two schemes are as efficient as each other. As $\delta_{\mathrm{rp}}$ increases so that the flow enters the transition and near-continuum regimes, the error in the CIS oscillates several times before it decays monotonically. As a consequence, the iteration number of CIS increases rapidly with the rarefaction parameter, which nearly scales as $\delta_{\mathrm{rp}}^{2}$. For the GSIS, however, the error is monotonically decreasing, and the rarefaction parameter does not influence the error decay rate, where the converged solutions are obtained within the same number of iterations (here 20 iterations) for each rarefaction parameter from the free molecular to continuum flow regimes. At $\delta_{\mathrm{rp}}=50$, the GSIS is about 100 times more efficient than the CIS, and it can be expected that the gain of using GSIS becomes larger and larger as $\delta_{\mathrm{rp}}$ further increases.

Another important property of the GSIS is that the numerical error caused by the spatial discretization is largely reduced when compared to that of the CIS. From Fig. 6 we see that when $N_{2}$ is decreased from 251 to 6 , that is, when the spatial cell size is respectively about $1 / 10$ and 5 times of the mean free path of gas molecules, the relative error in the density profile increases from $0.3 \%$ to $9 \%$, while that in the heat flux increases from $0.3 \%$ to $16 \%$ in the CIS. However, the relative error in the GSIS always remain within $1 \%$, even when the cell size is about 5 times larger than the gas mean free path. Note that even when $\delta_{\text {rp }}=500$, the heat flux obtained from the GSIS only changes from $3.721 \times 10^{-3}$ when $N_{2}=551$ to $3.726 \times 10^{-3}$ when $N_{2}=6$. The reason for this excellent performance is that the GSIS is asymptotically preserving the Navier-Stokes limit, while in the CIS the "numerical" thermal conductivity may be different to the physical one. Besides, in the CIS, the false convergence, e.g. the non-uniform distribution of heat flux in Fig. 6(b), may be reached when the spatial 


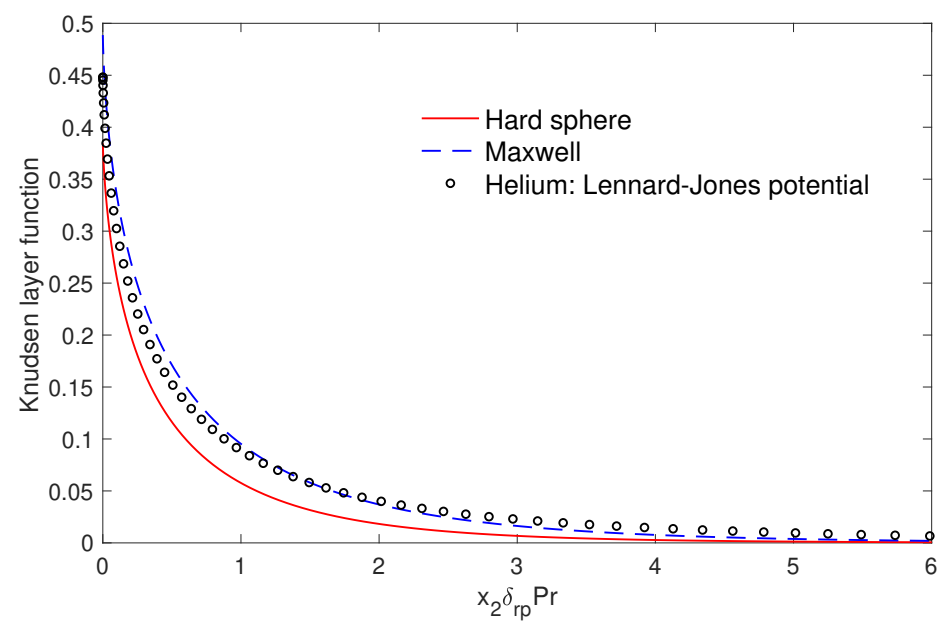

Figure 7: The Knudsen layer function $T_{s}$ for the temperature profile in the Fourier flow between two parallel plates obtained from GSIS. Note that the Lennard-Jones potential for helium is used at $T_{0}=300 \mathrm{~K}$, where the fast spectral method developed in Ref. [41] is used to calculate the Boltzmann collision operator.

resolution is not enough. The superior GSIS, however, does not suffer this problem.

It should be noted that the implicit UGKS [14] and other variants [45, 46] can also produce accurate results when the cell size is much larger than the molecular mean free path. This is achieved through a complex evaluation of the numerical flux at the cell interface to spontaneously treat the molecular streaming and collision. The GSIS, however, does not need complex flux evaluation since the Navier-Stokes constitutive laws are recovered explicitly.

Using the accurate and efficient GSIS, the LBE is solved for different molecular collision models (6) and the corresponding Knudsen layer functions are obtained. In the numerical simulation, we set the rarefaction parameter to be $\delta_{\mathrm{rp}}=60$, so that the distance between two plates is about 60 times as large as the mean free path of gas molecules; thus, the interference between the Knudsen layers near each plate is avoided. In the fast spectral approximation of the linearized Boltzmann collision operator (4), the integral with respect to the solid angle $\Omega$ is calculated by the Gauss-Legendre quadrature with $M=6$, see Eq. (39) in Ref. [40]. In the spatial discretization we set

$$
x_{2}=\left(10-15 s+6 s^{2}\right) s^{3}, \quad s=\left(0,1, \cdots, N_{\mathrm{s}}-1\right) / 2\left(N_{\mathrm{s}}-1\right)
$$

with $N_{\mathrm{s}}=200$. The iteration is terminated when $\epsilon<10^{-6}$.

When the steady-state solution is obtained, the temperature profile in the bulk region (i.e. $\left.0.4 \leq x_{2} \leq 0.5\right)$ is linearly fitted by $T_{\mathrm{NS}}=k_{1}\left(x_{2}-1 / 2\right)$ in the dimensionless form, where $k_{1}$ is the coefficient from the least square fitting of temperature. Then the Knudsen layer function is calculated according to the following equation:

$$
T_{s}\left(x_{2} \delta_{\mathrm{rp}} \operatorname{Pr}\right)=\delta_{\mathrm{rp}} \operatorname{Pr} \frac{T_{\mathrm{NS}}\left(x_{2}\right)-T\left(x_{2}\right)}{k_{1}}
$$


and the temperature jump coefficient is calculated as [56]

$$
\zeta_{T}=\frac{\delta_{\mathrm{rp}}}{2}\left(\frac{5}{4 \delta_{\mathrm{rp}} \operatorname{Pr}\left|q_{2}\right|}-1\right)
$$

When the LBE for HS, Helium and Maxwell molecules is solved by the GSIS, steadystate solutions are reached after 22, 25 and 27 iterations, respectively, and the temperature jump coefficients are respectively $1.892,1.933$ and 1.954 , which do not differ a lot between the three collision models. However, the Knudsen layer functions shown in Fig. 7 have larger difference. It is amazing that the small terms $2 \int\left(L-L_{\mathrm{s}}\right) v_{i} v_{j} \mathrm{~d} \boldsymbol{v}$ in Eq. (17) and $\int\left(L-L_{\mathrm{s}}\right) v_{i}|\boldsymbol{v}|^{2} \mathrm{~d} \boldsymbol{v}$ in Eq. (20) significantly affect the Knudsen layer function.

\subsection{Oscillatory Couette flow between two parallel plates}

Consider the rarefied gas dynamics between two infinite parallel plates with a distance $H$, located at $x_{2}=0$ and $x_{2}=1$. Both plates have a temperature $T_{0}$, the one at $x_{2}=1$ is stationary, while that at $x_{2}=0$ oscillating in the $x_{1}$ direction with the velocity

$$
U_{\mathrm{w}, 1}=\Re\left[\frac{U_{0}}{v_{\mathrm{m}}} \exp (i \mathrm{St} t)\right] .
$$

The Boltzmann equation is linearized by choosing $\alpha=U_{0} / v_{\mathrm{m}}$ in Eq. (25). If we consider the diffuse boundary condition, then we have $h\left(x_{2}=0, \boldsymbol{v}\right)=2 v_{1} f_{\mathrm{eq}}$ when $v_{2}>0$, and $h\left(x_{2}=1, \boldsymbol{v}\right)=0$ when $v_{2}<0$ [50]. The synthetic equations (13), (17), and (20) can be simplified to

$$
\begin{array}{r}
2 i \mathrm{St} U_{1}+\frac{\partial \sigma_{12}}{\partial x_{2}}=0, \\
i \mathrm{St} \sigma_{12}+\mathrm{HoT}_{\sigma_{12}}+\frac{\partial U_{1}}{\partial x_{2}}=-\delta_{\mathrm{rp}} \sigma_{12}+2 \int\left(L-L_{\mathrm{s}}\right) v_{1} v_{2} \mathrm{~d} \boldsymbol{v},
\end{array}
$$

where the moments involving even orders of $v_{1}$ are all zero, and we do not consider the heat flux $q_{1}$ in this problem as it does not affect the rate of convergence. It is noted that the above equations reduce to the synthetic equation developed in Ref. [33] when $\mathrm{St}=0$.

The two equations in Eq. (48) can be combined to produce the following diffusion equation for the flow velocity $U_{1}$ in the $(k+1)$-th iteration step:

$$
2 i \mathrm{St}\left(i \mathrm{St}+\delta_{\mathrm{rp}}\right) U_{1}^{(k+1)}-\frac{\partial^{2} U_{1}^{(k+1)}}{\partial x_{2}{ }^{2}}=\frac{\partial}{\partial x_{2}}\left[2 \int\left(L^{(k)}-L_{\mathrm{s}}^{(k)}\right) v_{1} v_{2} \mathrm{~d} \boldsymbol{v}-\mathrm{HoT}_{\sigma_{12}}^{(k)}\right] .
$$

In the numerical simulation, the spatial space is discretized by Eq. (44) with $N_{\mathrm{s}}=100$. The kinetic equation 22 is solved by the second-order upwind scheme, while the derivative in Eq. (49) is approximated by the central finite difference scheme with 5 stencils, and the resulting linear algebraic system for $U_{1}$ is solved exactly in the bulk region (i.e. at least three spatial points away from the boundary) in matrix form.

The comparison in accuracy and efficiency between the CIS and GSIS is summarized in Fig. 8, where the molecular velocity space is discretized in the same way as that in the 


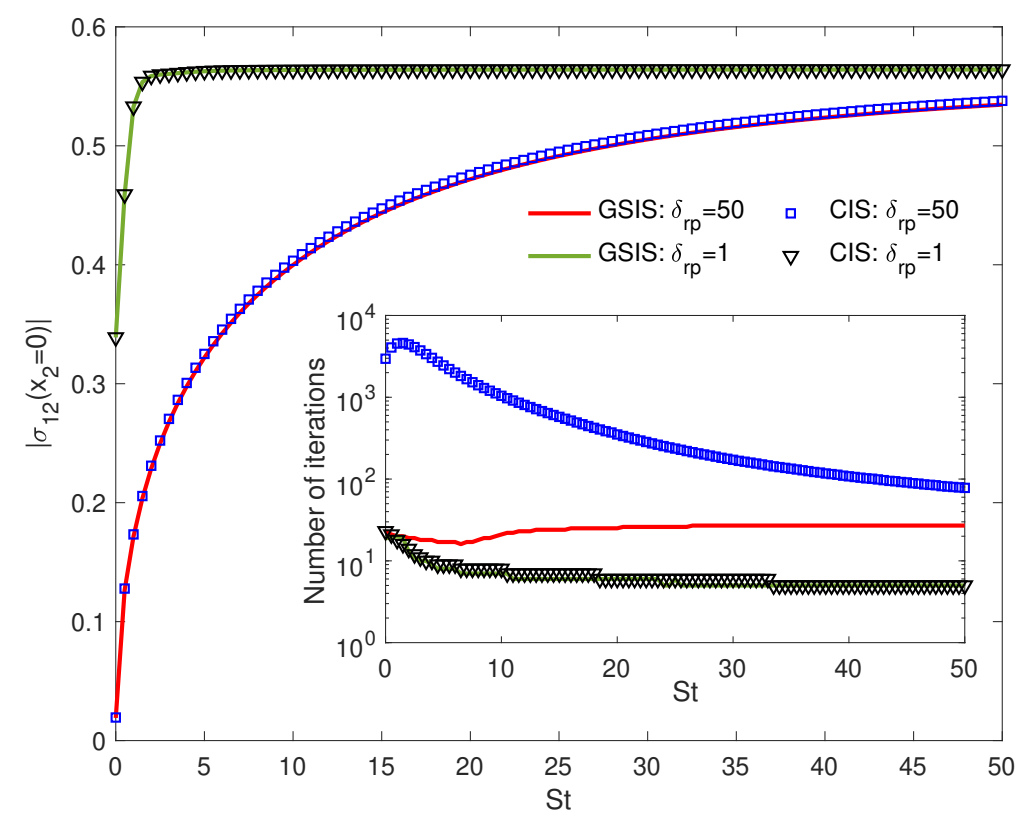

Figure 8: Comparisons of the amplitude of the shear stress exerting on the oscillating plate and iteration numbers (inset) between the CIS and GSIS, for the oscillating Couette flow. The Shakhov model is solved, where the solution is converged when $\int\left|\frac{U_{1}^{(k+1)}}{U_{1}^{(k)}}-1\right| \mathrm{d} x_{2}<10^{-5}$.

previous tests, but with $N_{\mathrm{v}}=96$ in Eq. (42). The relative difference in the amplitude of the shear stress $\sigma_{12}$ is within $1 \%$. When the rarefaction parameter is $\delta_{\mathrm{rp}}=50$, we see that the number of iterations in the CIS decreases from 30,000 to 100 when Strouhal number increases from 0 to 50. The reason for this reduction can be understood in the following way. The temporal Knudsen number $\mathrm{Kn}_{\mathrm{t}}$, which is defined as the ratio of characteristic oscillation frequency to the mean collision frequency of gas molecules, i.e.

$$
\mathrm{Kn}_{\mathrm{t}}=\frac{\varpi}{v_{\mathrm{m}} / \lambda}=\frac{\mathrm{St}}{\delta_{\mathrm{rp}}}
$$

increases with St. Therefore, even when $\delta_{\mathrm{rp}}$ is large, that is, when the spatial Knudsen number is small, the large temporal Knudsen number can also make the flow rarefied, and the more rarefied the gas, the fast the iteration to the steady-state. Even with this effect, the GSIS is still faster than the CIS: only about 20 iterations are needed in the GSIS for each Strouhal number considered.

However, for the GSIS in oscillating problems, there is a problem, like the one encountered in Sec. 4. From Eq. (49) we see that the eigenvalue of this second-order differential equation is imaginary, which means that when $\delta_{\mathrm{rp}}$ is small and St is large, the solution will change quasi-periodically in the spatial direction with large frequency, whereas physically the solution should decay fast from the oscillating sources as the dissipation is huge due to the large values of both spatial and temporal Knudsen numbers. Mathematically speaking, for highly oscillating solutions, any slight inaccurate boundary conditions will lead to com- 
pletely different solutions. Therefore, in the numerical simulation, when we solve Eq. 49 directly, the solution is either wrong or blows up. To fix this problem, again we introduce a relative large value of $\bar{\delta}_{\text {rp }}$ to decay the fast oscillation. That is, instead of solving Eq. (49), we solve the following diffuse-type equation:

$$
\begin{aligned}
2 i \operatorname{St}\left(i \mathrm{St}+\bar{\delta}_{\mathrm{rp}}\right) U_{1}^{(k+1)}-\frac{\partial^{2} U_{1}^{(k+1)}}{\partial x_{2}{ }^{2}}= & \frac{\partial}{\partial x_{2}}\left[2 \int\left(L^{(k)}-L_{\mathrm{s}}^{(k)}\right) v_{1} v_{2} \mathrm{~d} \boldsymbol{v}-\mathrm{HoT}_{\sigma_{12}}^{(k)}\right] \\
& +2 i \operatorname{St}\left(\bar{\delta}_{\mathrm{rp}}-\delta_{\mathrm{rp}}\right) U_{1}^{(k+1 / 2)}
\end{aligned}
$$

where

$$
\bar{\delta}_{\mathrm{rp}}=\max \left(\delta_{\mathrm{rp}}, \mathrm{St}\right) \text {. }
$$

It can be proven that, when the solution of Eq. (51) converges, Eqs. (51) and (49) are equivalent. This treatment does not affect the accuracy and efficiency of the GSIS when $\delta_{\text {rp }}$ is small, while when $\delta_{\mathrm{rp}}$ is large, the solution from the synthetic equations is always stable, and we see in Fig. 8 that in most cases the GSIS needs slightly less iterations than CIS.

In addition to the significant reduction of iteration number, the GSIS needs less spatial grids than that of the CIS. Two examples are given in Fig. 9, where one can see that the GSIS can yield accurate results even when the cell sizes are respectively about 6.6 and 50 times of the molecular mean free path, while the CIS has large error due to the strong numerical dissipation for unresolved spatial cells.

\subsection{Sound propagation between two parallel plates}

Consider the sound propagation through a gas between two infinite parallel plates with a distance $H$, located at $x_{2}=0$ and $x_{2}=1$. The two plates have a temperature $T_{0}$, the one at $x_{2}=1$ is stationary, while that $x_{2}=0$ oscillating in the $x_{2}$ direction with the speed $U_{\mathrm{w}, 2}=\Re\left[\left(U_{0} / v_{\mathrm{m}}\right) \exp (i \mathrm{St} t)\right]$. The Boltzmann equation is linearized by choosing $\alpha=U_{0} / v_{\mathrm{m}}$ in Eq. (25). The boundary conditions are [51]

$$
\begin{aligned}
& h\left(x_{2}=0, \boldsymbol{v}\right)=\left[\sqrt{\pi}+2 v_{2}-2 \sqrt{\pi} \int_{v_{2}<0} v_{2} h\left(x_{2}=0, \boldsymbol{v}\right) \mathrm{d} \boldsymbol{v}\right] f_{\text {eq }}, \quad \text { when } v_{2}>0, \\
& h\left(x_{2}=1, \boldsymbol{v}\right)=2 \sqrt{\pi} f_{\text {eq }} \int_{v_{2}<0} v_{2} h\left(x_{2}=1, \boldsymbol{v}\right) \mathrm{d} \boldsymbol{v}, \quad \text { when } v_{2}<0 .
\end{aligned}
$$

The synthetic equations (13), (17), and 20) can be simplified to

$$
\begin{array}{r}
i \mathrm{St} \rho+\frac{\partial U_{2}}{\partial x_{2}}=0, \\
2 i \operatorname{St} U_{2}+\frac{\partial \rho}{\partial x_{2}}+\frac{\partial T}{\partial x_{2}}+\frac{\partial \sigma_{22}}{\partial x_{2}}=0, \\
\frac{3}{2} i \operatorname{St} T+\frac{\partial q_{2}}{\partial x_{2}}+\frac{\partial U_{2}}{\partial x_{2}}=0, \\
i \operatorname{St}_{22}+\operatorname{HoT}_{\sigma_{22}}+\frac{4}{3} \frac{\partial U_{2}}{\partial x_{2}}=-\delta_{\mathrm{rp}} \sigma_{22}+2 \int\left(L-L_{\mathrm{s}}\right)\left(v_{2}^{2}-\frac{|\boldsymbol{v}|^{2}}{3}\right) \mathrm{d} \boldsymbol{v}, \\
i \mathrm{St}_{2}+\mathrm{HoT}_{q_{2}}+\frac{3 C_{q}}{2} \frac{\partial T}{\partial x_{2}}=-\frac{2}{3} \delta_{\mathrm{rp}} q_{2}+\int\left(L-L_{\mathrm{s}}\right) v_{2}|\boldsymbol{v}|^{2} \mathrm{~d} \boldsymbol{v} .
\end{array}
$$



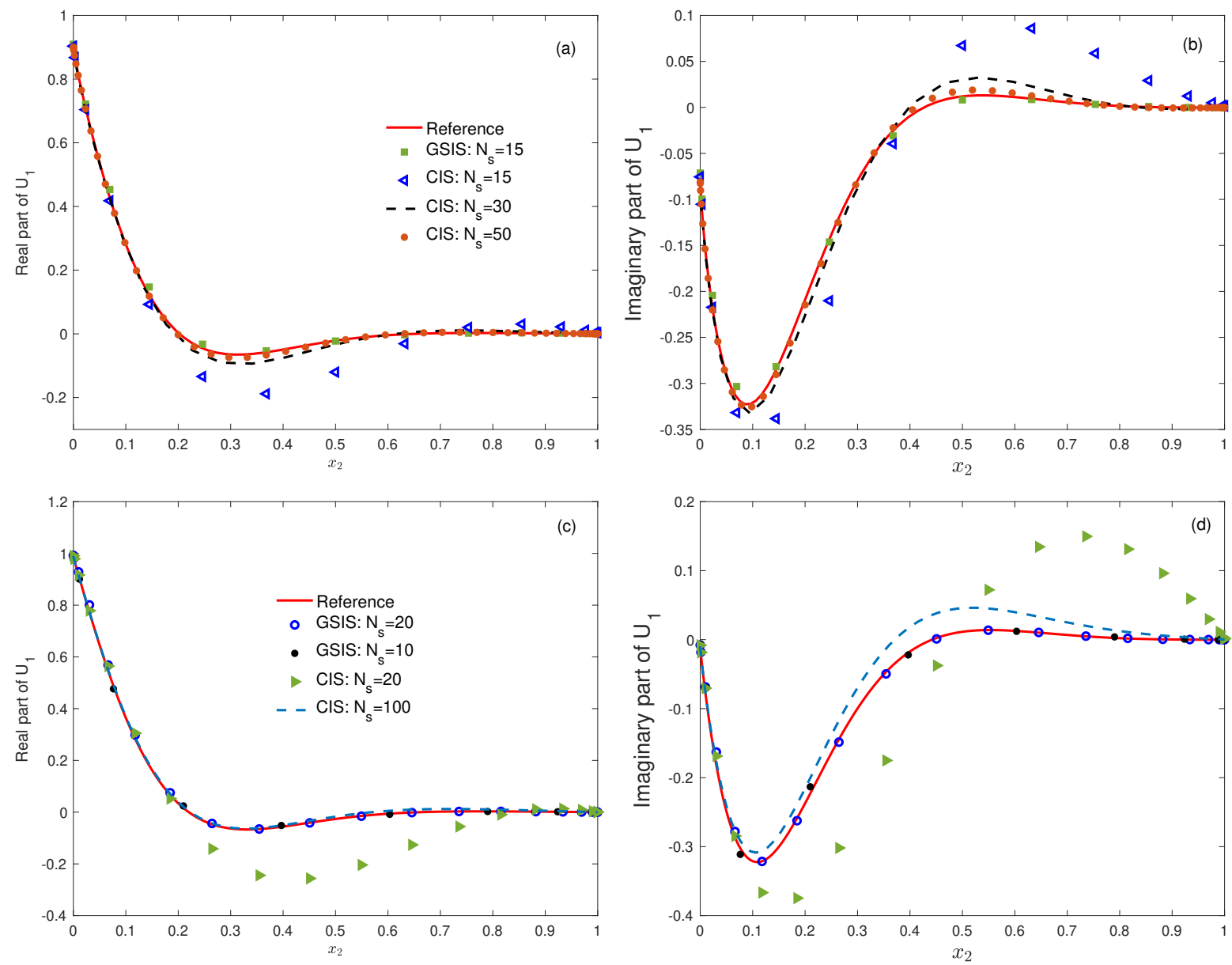

Figure 9: Comparisons of the velocity profiles in the oscillating Couette flow with different spatial discretizations, when $(\mathrm{a}, \mathrm{b}) \delta_{\mathrm{rp}}=50$ and $\mathrm{St}=1$ and $(\mathrm{c}, \mathrm{d}) \delta_{\mathrm{rp}}=500$ and $\mathrm{St}=0.1$. The reference solution is obtained from the GSIS, where the spatial domain is discretized by Eq. 444, with $N_{\mathrm{s}}=100$ when $\delta_{\mathrm{rp}}=50$ and $N_{\mathrm{s}}=500$ when $\delta_{\mathrm{rp}}=500$.

These synthetic equations can be combined to form two diffusion equations for the flow velocity $U_{2}$ and temperature $T$. To quickly decay the non-physical oscillations when $\delta_{\mathrm{rp}}$ is small and St is large, in numerical iterations we set

$$
\begin{aligned}
& \delta_{\mathrm{rp}} \sigma_{22}^{(k+1)}=\bar{\delta}_{\mathrm{rp}} \sigma_{22}^{(k+1)}+\left(\delta_{\mathrm{rp}}-\bar{\delta}_{\mathrm{rp}}\right) \sigma_{22}^{(k+1 / 2)}, \\
& \delta_{\mathrm{rp}} q_{2}^{(k+1)}=\bar{\delta}_{\mathrm{rp}} q_{2}^{(k+1)}+\left(\delta_{\mathrm{rp}}-\bar{\delta}_{\mathrm{rp}}\right) q_{2}^{(k+1 / 2)},
\end{aligned}
$$

where $\bar{\delta}_{\text {rp }}$ is given in Eq. (52). When $U_{2}$ and $T$ are solved, the perturbed density, shear stress and heat flux can be solved from Eqs. (54), (57), and (58).

Typical numerical results are shown in Fig. 10 when the spatial region $x_{2} \in[0,1]$ is discretized by 200 uniformly-distributed points, while the velocity grids are the same as that used in Sec. 5.2. For the CIS, it is very hard to find the converged solution when the Strouhal number St is small, where the iteration number scales roughly as $\mathrm{St}^{-1.5}$. However, this 

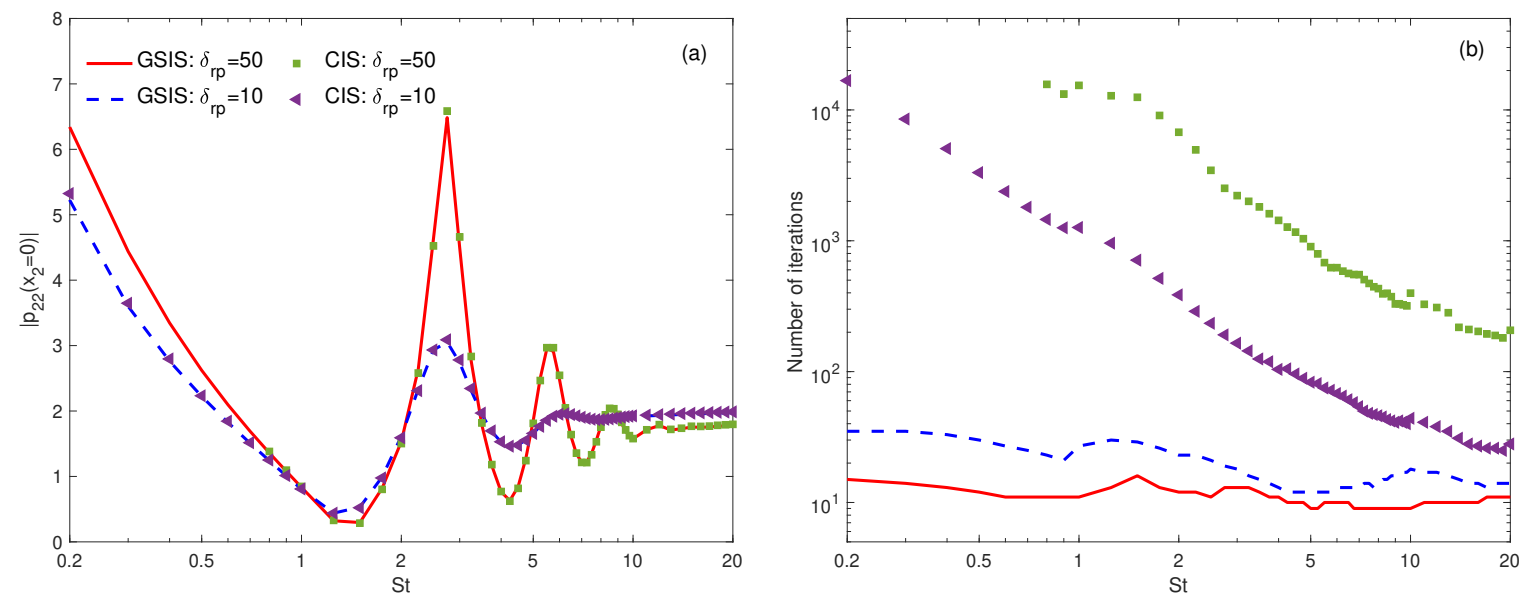

Figure 10: Comparisons of (a) the amplitude of normal pressure exerting on the oscillating plate and iteration numbers (b) between the CIS and GSIS, for the sound propagation problem. The Shakhov model is solved, where the solution is converged when $\max \left\{\int\left|\frac{\rho^{(k+1)}}{\rho^{(k)}}-1\right| \mathrm{d} x_{2}, \int\left|\frac{U_{2}^{(k+1)}}{U_{2}^{(k)}}-1\right| \mathrm{d} x_{2}, \int\left|\frac{T^{(k+1)}}{q^{(k)}}-1\right| \mathrm{d} x_{2}\right\}<$ $10^{-5}$.

problem does not exist in the GSIS, as the Strouhal number has little effect on the number of iterations. The effect of spatial resolution on the fidelity of the solution is demonstrated in Fig. 11 when $\mathrm{St}=2.5$, where the sound waves between two plates have resonance. It is seen that the GSIS needs less spatial grids than CIS. Again, this example proves the accuracy and efficiency of the GSIS.

\section{Numerical tests for two-dimensional problems}

Now we consider two-dimensional problems such as lid-driven cavity flow and sheardriven flow between two eccentric cylinders. The problems are not able to be simulated by the special SIS only applicable for rarefied gas flows [25, 30, 31, 27, 43, 33], where the flow velocity is perpendicular to the computational domain. Here we investigate the performance of GSIS for typical general rarefied gas flows, where the flow velocity (or other macroscopic variables) varies within the computational domain.

\subsection{Two-dimensional lid-driven cavity flow}

The two-dimensional lid-driven cavity flow is a canonical test for the algorithms for both the Navier-Stokes and gas-kinetic equations. The flow domain is a square with size of $1 \times 1$, with the left and right walls locate at $x_{1}=0$ and $x_{1}=1$, bottom and top walls at $x_{2}=0$ and $x_{2}=1$, respectively. The top wall moves in the $x_{1}$ direction with a constant velocity of $U_{\mathrm{w}}$, while the other walls are static. All the walls are kept at uniform temperature $T_{0}$. To demonstrate the accuracy and efficient of the GSIS, the Shakhov kinetic equation is 

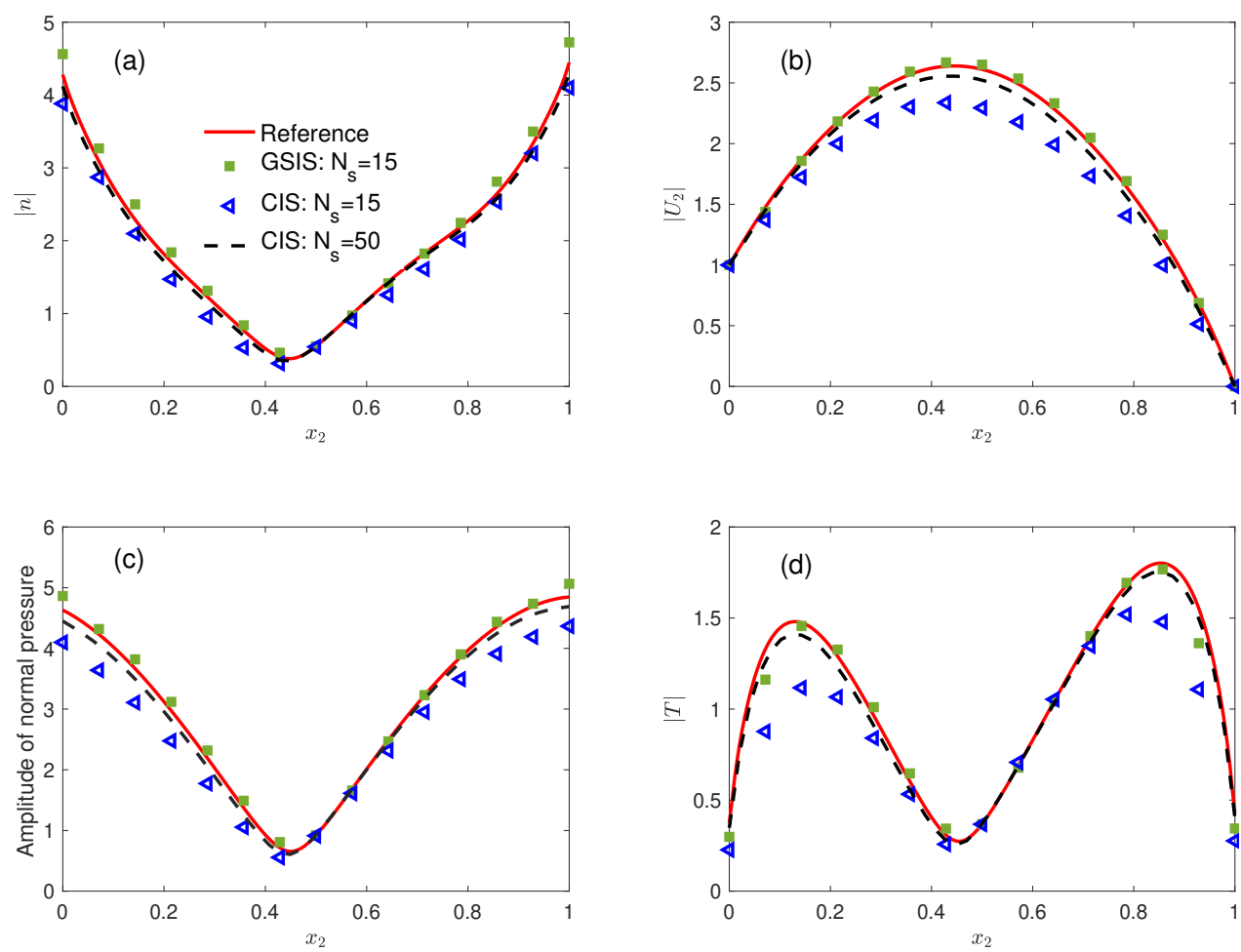

Figure 11: Profiles of macroscopic quantities in the sound propagation problem with different spatial discretizations, when $\delta_{\mathrm{rp}}=50$ and $\mathrm{St}=2.5$. The reference solution is obtained from the GSIS, where the spatial domain is discretized by $N_{\mathrm{s}}=200$ uniform grids; the corresponding CIS results overlap with these lines and are not shown here. The normal pressure is defined as $P_{22}=2 \int v_{2}^{2} h \mathrm{~d} \boldsymbol{v}$.

linearized by choosing $\alpha=U_{\mathrm{w}} / v_{\mathrm{m}}$ in Eq. (2). The boundary conditions are

$$
\begin{aligned}
& h\left(x_{1}=0, \boldsymbol{v}\right)=-2 \sqrt{\pi} f_{\text {eq }} \int_{v_{1}<0} v_{1} h\left(x_{1}=0, \boldsymbol{v}\right) \mathrm{d} \boldsymbol{v}, \quad \text { when } v_{1}>0, \\
& h\left(x_{1}=1, \boldsymbol{v}\right)=2 \sqrt{\pi} f_{\text {eq }} \int_{v_{1}>0} v_{1} h\left(x_{1}=1, \boldsymbol{v}\right) \mathrm{d} \boldsymbol{v}, \quad \text { when } v_{1}<0, \\
& h\left(x_{2}=0, \boldsymbol{v}\right)=-2 \sqrt{\pi} f_{\text {eq }} \int_{v_{2}<0} v_{2} h\left(x_{2}=0, \boldsymbol{v}\right) \mathrm{d} \boldsymbol{v}, \quad \text { when } v_{2}>0, \\
& h\left(x_{2}=1, \boldsymbol{v}\right)=\left[2 v_{1}+2 \sqrt{\pi} \int_{v_{2}>0} v_{2} h\left(x_{2}=1, \boldsymbol{v}\right) \mathrm{d} \boldsymbol{v}\right] f_{\text {eq }}, \quad \text { when } v_{2}<0 .
\end{aligned}
$$

The problem is solved on non-uniform Cartesian grids, where dimensions in both the axes $x_{1}$ and $x_{2}$ are discretized by Eq.(44). The linearized Shakhov equation is solved by DVM with the 2nd-order upwind finite-difference scheme, where the distribution functions are stored at the centers of grid cells. In GSIS, Eq. (13) with the constitutive relations in Eqs. (17) and (20) lead to the Navier-Stokes-Fourier equations with source terms; these equations are solved using a finite-difference version of the Semi-Implicit Method for Pressure Linked Equations (SIMPLE). In each SIMPLE iteration, we solve four discrete diffusive equations 


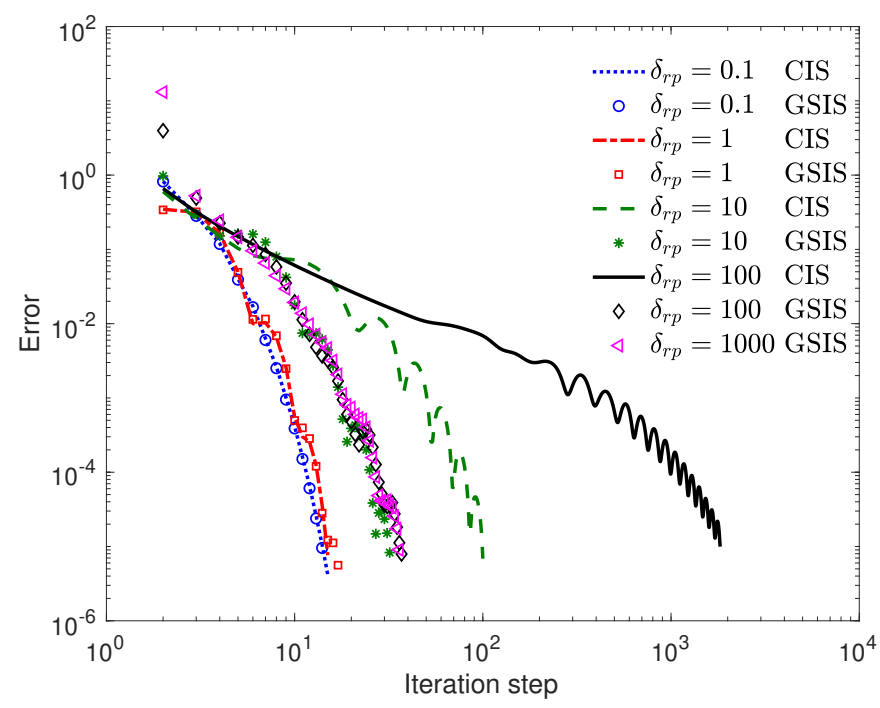

Figure 12: The decay of error $\epsilon$ as a function of the iteration step in the lid-driven cavity flow described by the linearized Shakhov model. The iteration is assumed to be converged when $\epsilon$ defined in Eq. 62 is less than $10^{-5}$.

(for the two velocity components, pressure correction, and temperature) using the Jacobi iteration methods.

After the macroscopic flow variables are solved by SIMPLE algorithm, the velocity distribution function is updated as

$$
h^{(k+1)}(\boldsymbol{x}, \boldsymbol{v})=h^{(k+1 / 2)}(\boldsymbol{x}, \boldsymbol{v})+\frac{\delta_{\mathrm{rp}}}{\max \left(10, \delta_{\mathrm{rp}}\right)}\left[\lambda_{\rho}(\boldsymbol{x})+2 \lambda_{\boldsymbol{U}}(\boldsymbol{x}) \cdot \boldsymbol{v}+\lambda_{T}(\boldsymbol{x})\left(|\boldsymbol{v}|^{2}-\frac{3}{2}\right)\right] f_{\mathrm{eq}},
$$

because (i) the update of the shear stress and heat flux does not affect the accuracy and efficiency of the GSIS, and (ii) for highly rarefied gas flows, high-order terms are very large and the macroscopic synthetic equations become stiff near the solid corners due to the small value of $\delta_{\mathrm{rp}}$, hence the limiter $\delta_{\mathrm{rp}} / \max \left(10, \delta_{\mathrm{rp}}\right)$ is introduced to retain the numerical stability.

We first test the converging speeds of both CIS and GSIS for the cases of $\delta_{\mathrm{rp}}=0.1,1$, 10, 100 and 1000. The corresponding spatial grids are non-uniform with $N_{\mathrm{s}}=21,21,21,41$, 61 respectively. For the cases of $\delta_{\mathrm{rp}}=0.1,1$ and 10, the molecular velocity in both $v_{1}$ and $v_{2}$ are discretized by Eq. (42), with $\imath=3$, and $N_{\mathrm{v}}=48,48$ and 24, respectively, while for $v_{3}$, 24,24 and 12 uniform points in the range of $[-6,6]$ are used, respectively. When $\delta=100$ and 1000, the 6- and 8-point Gauss-Hermite quadrature nodes are used in all three velocity components. The iterations in both CIS and GSIS are assumed to be converged when

$$
\epsilon=\iint\left|\frac{\left|\boldsymbol{U}^{(k+1)}\right|}{\left|\boldsymbol{U}^{(k)}\right|}-1\right| \mathrm{d} x_{1} \mathrm{~d} x_{2}<10^{-5} .
$$

The same criterion is used for the inner loop of SIMPLE algorithm for the Navier-Stokes equations. 
Table 1: Number of iteration steps and CPU time to reach convergence for the lid-driven cavity flow.

\begin{tabular}{rllrrrr}
\hline$\delta_{\text {rp }}$ & $N_{\mathrm{s}}^{2}$ & $N_{v_{1}} N_{v_{2}} N_{v_{3}}$ & \multicolumn{2}{c}{ Iteration steps } & \multicolumn{2}{c}{ Total CPU time (s) } \\
& & & CIS & GSIS & CIS & GSIS \\
\hline 0.1 & $20 \times 20$ & $48 \times 48 \times 24$ & 14 & 13 & 28.5 & 32.6 \\
1 & $20 \times 20$ & $48 \times 48 \times 24$ & 14 & 16 & 28.2 & 38.4 \\
10 & $20 \times 20$ & $24 \times 24 \times 12$ & 99 & 31 & 121.4 & 47.7 \\
100 & $40 \times 40$ & $16 \times 16 \times 16$ & 1823 & 36 & 3176.1 & 144.1 \\
1000 & $60 \times 60$ & $8 \times 8 \times 8$ & - & 36 & - & 492.5 \\
\hline
\end{tabular}

Figure 12 compares the decay of error $\epsilon$ as a function of the number of iteration steps in CIS and GSIS for flows at different values of rarefaction parameter, while Table 1 summarizes the number of iteration steps and the total CPU time, where the single threaded Matlab 2018 code is run on Intel Xeon-E5-2680 v4 CPU. Similar to the test case of the Fourier heat transfer, at small values of $\delta_{\mathrm{rp}}(0.1$ and 1$)$, errors in both GSIS and CIS decay with the same rate and converged solutions are found within 20 iteration steps. In the cases of larger $\delta_{\mathrm{rp}}$, the iteration step in GSIS slightly increases, but it is less than 40 steps even for the case of $\delta_{\mathrm{rp}}=1000$. In contrast, the convergence of the CIS iteration deteriorates severely as $\delta_{\mathrm{rp}}$ increases. The iteration step reaches 1823 for the case of $\delta_{\mathrm{rp}}=100$. Due to the slow convergence of CIS for near continuum flows, the case of $\delta_{\mathrm{rp}}=1000$ is not simulated.

With significantly faster convergence rate, the GSIS takes much less CPU time than the CIS for cases of large $\delta_{\text {rp }}$ as shown in Table 1. Note that although the iteration number is reduced in GSIS, the time for each iteration increases as the cost to solve the synthetic equations is non-negligible, see the last column of Table 1. This is because the inner loop of the SIMPLE algorithm based on segregated approach can take up to several hundreds iterations to converge, depending on the value of $\delta_{\mathrm{rp}}$. The iteration steps of each inner loop to reach convergence is dynamically decreasing with the DVM (outer) iteration, and settles down to a constant which is rarefication-parameter related, e.g., from 677 to 155 in the case of $\delta_{\mathrm{rp}}=100$, while 178 to 76 in the case of $\delta_{\mathrm{rp}}=10$. We note that using a coupled algorithm to solve the discretized pressure and velocity components in a single linear equation system would be much faster than the segregated approach, especially for high $\delta_{\mathrm{rp}}$ cases, as have been studied in the incompressible CFD theories. For example, in the following section we find that if the kinetic and synthetic equations are both solved by DG, the cost of DG for synthetic equations is negligible since pressure, velocity, and temperature are solved simultaneously by direct solver.

To compare the accuracy of the GSIS with the CIS, we simulated the case of $\delta_{\mathrm{rp}}=100$ with different non-uniform physical grids, including $N_{\mathrm{s}}=21,41,61$ and 101. Fig. 13 compares the pressure fields and streamlines predicted by both the CIS and GSIS, in which the reference solutions are chosen as the GSIS results on the grid of $N_{\mathrm{s}}=61$. It can be seen that the GSIS solution on the coarsest grid $\left(N_{\mathrm{s}}=21\right)$ is much more accurate than the CIS counterpart, especially in terms of the pressure field. From Fig. 13(d) to (f), we observe that the short contour lines near the bottom wall are accurately captured by the GSIS even on the coarsest mesh, while the CIS can capture them only with the finest mesh, see Fig. 13 


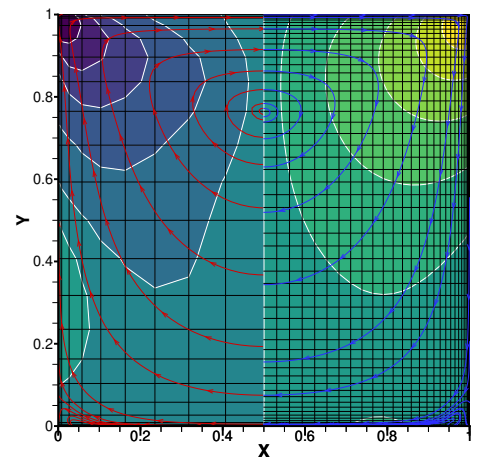

(a) CIS $N_{s}=21$

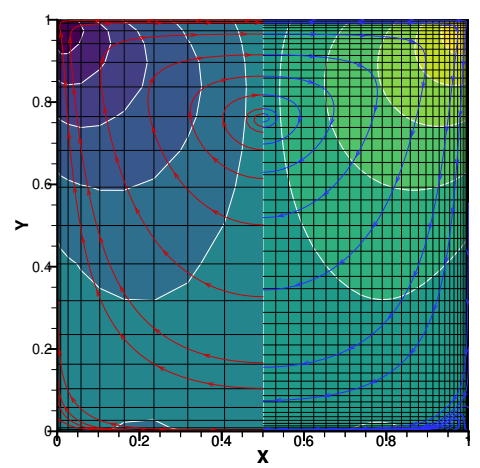

(d) GSIS $N_{s}=21$

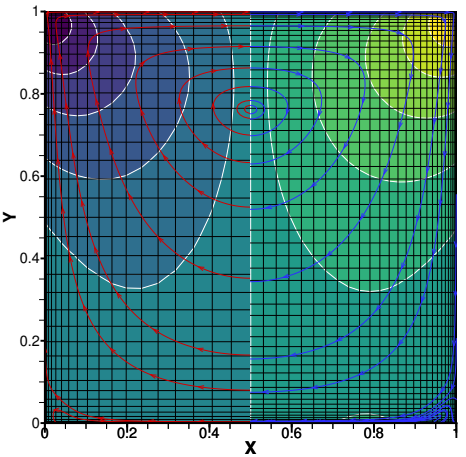

(b) CIS $N_{s}=41$

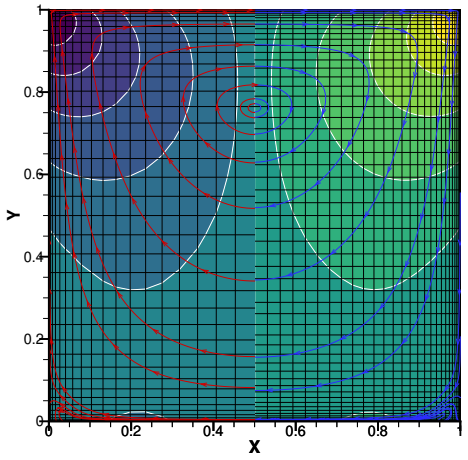

(e) GSIS $N_{s}=41$

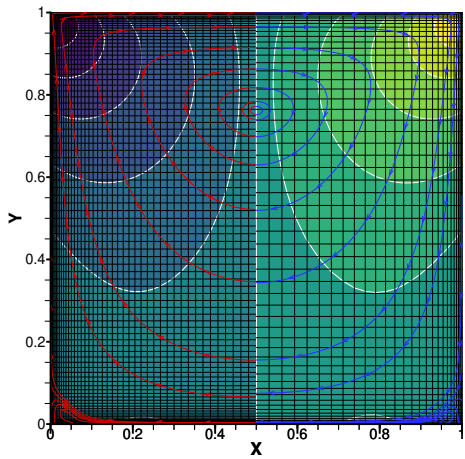

(c) CIS $N_{s}=101$

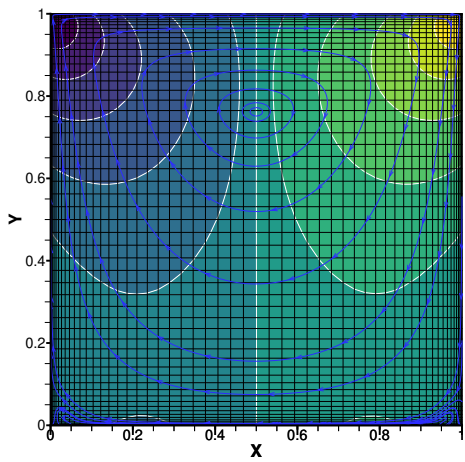

(f) GSIS $N_{s}=61$

Figure 13: Accuracy comparisons between the CIS and GSIS for the lid-driven cavity flow. In each plot, the right half are the reference solution (GSIS results on the $N_{\mathrm{s}}=61$ grid). In the upper rows, the left halves of the plots are CIS solutions on grids of $N_{\mathrm{s}}=21,41$ and 101 from left to right. In the lower rows, the left halves are GSIS solution on grids of $N_{\mathrm{s}}=21,41$ and 61 from lest to right. The contour plot is pressure $(\rho+T)$, with contour levels of $-0.2,-0.1,-0.05,-0.02,-0.005,0,0.005,0.02,0.05,0.1$ and 0.2 .

(c).

\subsection{Shear-driven flow between two eccentric cylinders}

In this section, we consider a shear-driven gas flow between two noncoaxial cylinders. This test case is used to show that the proposed GSIS can be efficiently implemented through other CFD method rather than the finite difference algorithm to deal with more complicated geometries. As shown in Fig. 14, the outer cylinder with a radius of 2 rotates clockwise at a constant speed of $U_{\mathrm{w}}$, while the inner cylinder with a radius of 1 keeps static. The centers of the outer cylinder and inner cylinder are at $\boldsymbol{x}=(0,0.5)$ and the origin, respectively. Both cylinders have a constant temperature $T_{0}$. It is assumed that $U_{\mathrm{w}}$ is much smaller than the most probable speed $v_{\mathrm{m}}$, thus the gas system can be linearized with $\alpha=U_{\mathrm{w}} / v_{\mathrm{m}}$. The velocity distribution function for reflected molecules at the outer cylinder is given by 


$$
h(\boldsymbol{x}, \boldsymbol{v})=\left[2 \boldsymbol{t}_{\mathrm{w}} \cdot \boldsymbol{v}-2 \sqrt{\pi} \int_{\boldsymbol{v}^{\prime} \cdot \boldsymbol{n}_{\mathrm{w}}<0} \boldsymbol{v}^{\prime} \cdot \boldsymbol{n}_{\mathrm{w}} h\left(\boldsymbol{x}, \boldsymbol{v}^{\prime}\right) \mathrm{d} \boldsymbol{v}^{\prime}\right] f_{\mathrm{eq}}, \quad \text { when } \boldsymbol{v} \cdot \boldsymbol{n}_{\mathrm{w}}>0,
$$

where $\boldsymbol{n}_{\mathrm{w}}$ and $\boldsymbol{t}_{\mathrm{w}}$ denote the outward unit normal vector and tangential vector of the solid surface. The boundary condition at the inner cylinder is similar but without the term $\boldsymbol{t}_{\mathrm{w}} \cdot \boldsymbol{v}$.

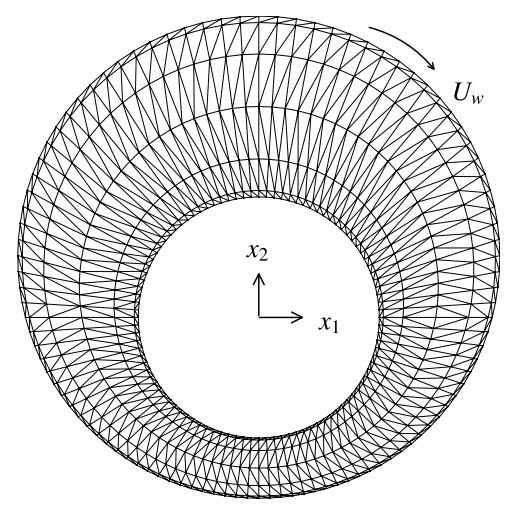

Figure 14: Schematic of the geometry and structured triangular mesh for shear-driven flow between two eccentric cylinders.

Using both the GSIS and CIS, the shear-driven flow is simulated on structured triangular mesh, in which the grid nodes along the radial direction is described by Eq. (44). The highorder DG methods are employed to seek solutions of the linearized Shakhov model equation and the synthetic macroscopic equations, in pecewise polynomial spaces of degree of 3 . The detailed DG scheme for the gas kinetic equation can be found in [44], while the hybridizable DG algorithm to solve the synthetic macroscopic equations is listed in the Appendix. During each iteration step, besides the $N_{\mathrm{v}}$ discretized kinetic equations that are solved successively on each spatial cell using the sweeping technique, one linear system (generated after DG discretization) of dimension $4(\mathcal{K}+1) N_{\mathrm{f}} \times 4(\mathcal{K}+1) N_{\mathrm{f}}$ for the evolutions of all the maroscopic unknowns over the whole computational domain is solved by the direct solver for large sparse linear system based on $L U$-decomposition. Here the number 4 represents the number of unknowns, i.e. $p, u_{1}, u_{2}$ and $T, \mathcal{K}$ is the degree of approximating polynomials in DG discretization and $N_{\mathrm{f}}$ is the number of faces in spatial mesh skeleton.

The resultant velocity contours and streamlines are illustrated in Fig. 15 for two selected rarefaction parameters $\delta_{\mathrm{rp}}=1000$ and 10, in which the GSIS solutions are plotted in the left half domain and the CIS ones are plotted in the right half domain. The results at $\delta_{\mathrm{rp}}=1000$ are obtained on 2400 triangles with cell size (characterized by the height of triangle) varying from 3 to 260 times the mean free path of gas molecules. The molecule velocity space is discretized by 8-point Gauss-Hermite quadrature nodes in $v_{1}$ and $v_{2}$ and 12 equidistant nodes in the range of $[-4,4]$ in $v_{3}$. The results at $\delta_{\mathrm{rp}}=10$ are obtained on 1600 triangles with cell size varying from 0.1 to 3 times the mean free path of gas molecules. The molecule velocity space is discretized in the domain of $[-4,4]^{3}$ by 32 non-uniform nodes in $v_{1}$ and $v_{2}$ 

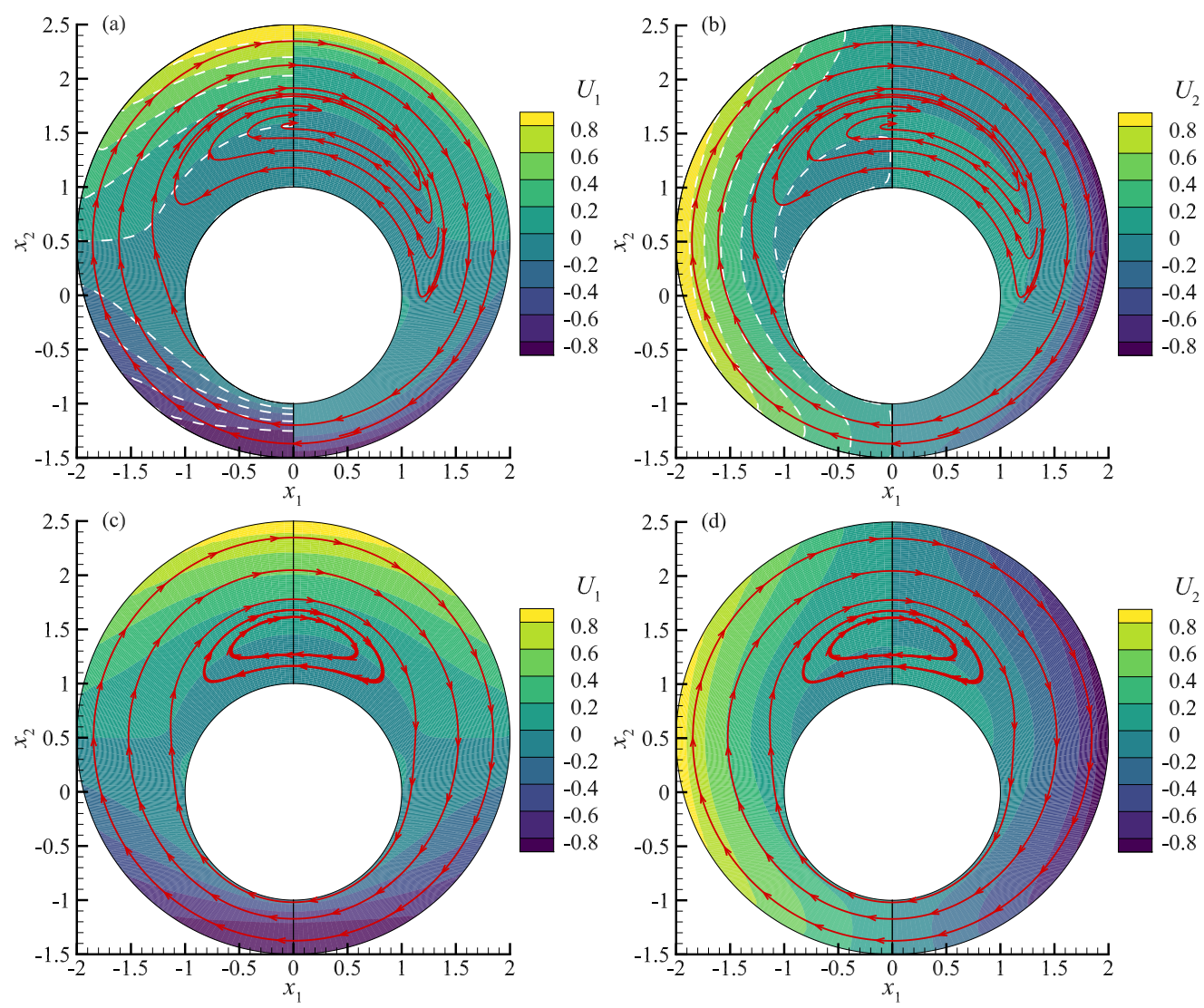

Figure 15: Comparisons of the CIS and GSIS results for shear-driven flow between two eccentric cylinders. (a) Contours of $U_{1}$ and streamlines at $\delta_{\mathrm{rp}}=1000$; (b) Contours of $U_{2}$ and streamlines at $\delta_{\mathrm{rp}}=1000$; (c) Contours of $U_{1}$ and streamlines at $\delta_{\mathrm{rp}}=10$; (d) Contours of $U_{2}$ and streamlines at $\delta_{\mathrm{rp}}=10$. In each sub-figures, the GSIS results are plotted in the left half domain while the CIS ones are illustrated in the right half domain. In (a) and (b) the velocity contours obtained by only solving the Navier-Stokes equations with non-slip velocity boundary are also included, which are indicated by the white dashed lines.

and 24 equidistant nodes in $v_{3}$. Numerical solutions are believed to be converged when the relative error in velocity magnitude $|\boldsymbol{U}|$ between two consecutive iteration steps is less than $10^{-5}$. The streamlines show that, as the gas rotates clockwise from the top to the bottom, due to the shrink of the flow pass, part of the gas near the outer surface is squeezed into the bottom narrow space while the other part of the gas flows back along the surface of the inner cylinder; as a consequence, a vortex appears above the inner cylinder.

Large discrepancies in the velocity contours are observed between the GSIS and CIS results at $\delta_{\mathrm{rp}}=1000$. To test the accuracy of both schemes, we also include the results of the Navier-Stokes equations with non-slip velocity boundary condition, which are illustrated by the white dashed lines in Fig. 15(a) and (b). The GSIS results overlap with the ones from the Navier-Stokes equations, thus the GSIS can asymptotically preserve the NavierStokes limit. However, the CIS cannot predict accurate solutions due to the large numerical dissipation on such a coarse mesh, i.e. the maximum cell size is about 260 times of the molecular mean free path. As the rarefaction parameter decreases to 10, the GSIS and CIS 
can produce close solutions on the same mesh.

Consider the rate of convergence to the steady-state solution, the numbers of iteration steps and CPU time to reach convergence for both the CIS and GSIS are listed in Table 2 . All the cases are done in double precision on Intel Xeon-E5-2680 processors and 128 GB RAM and the direct sparse solver, PARDISO [57] is called to directly solve the linear system for macroscopic equations. The simulations are run on 12 processors using OpenMP. It is seen that the GSIS cost only 26 iterative steps to reach the convergence criterion for both the cases of $\delta_{\mathrm{rp}}=1000$ and 10, while the CIS consumes 49454 and 296 steps, respectively. Compared to that of solving the kinetic equation, the computational consumption for DG to solve the macroscopic equations is very small, since the number of degrees of freedom for the latter one is much smaller. Therefore, the GSIS can be nearly 1300 and 5 times faster than the CIS when $\delta_{\mathrm{rp}}=1000$ and 10 , respectively.

Table 2: Number of iteration steps and CPU time to reach convergence for the shear-driven flow between two eccentric cylinders.

\begin{tabular}{rllrrrr}
\hline$\delta_{\text {rp }}$ & \# of triangles & $N_{v_{1}} N_{v_{2}} N_{v_{3}}$ & Iteration steps & \multicolumn{2}{c}{ Total CPU time (s) } \\
& & & CIS & GSIS & CIS & GSIS \\
\hline 1000 & 2400 & $8 \times 8 \times 12$ & 49454 & 26 & 33861.2 & 26.3 \\
10 & 1600 & $32 \times 32 \times 24$ & 296 & 26 & 2849.8 & 580.3 \\
\hline
\end{tabular}

\subsection{Two-dimensional oscillatory Couette flow}

Finally we consider the oscillatory flow in a three-dimensional cavity shown in Fig. 16(a). We assume the side length $\mathrm{OD}$ is much larger than $\mathrm{OH}$ and $\mathrm{OA}$, so that the problem is quasi two-dimensional. The characteristic length $H$ is chosen as the side length OA, and the aspect ratio is defined as $\mathrm{Asp}=O H / O A$. If $\mathrm{Asp}=\infty$, the problem is just the oscillatory Couette flow between two parallel plates studied in Sec. 5.2. This problem is interesting because it displays a counter-intuitive phenomenon that the shear force exerting on the oscillating lid in two-dimensional cavity could be even smaller than that of the one-dimensional Couette flow [52]. The full three-dimensional oscillatory flow was studied in Ref. [58], but not all the parameter region are covered, for example, the case with OA much larger than OA and $\mathrm{OH}$.

The synthetic equations (13), (17), and (20) can be simplified to

$$
\begin{array}{r}
2 i \operatorname{St} U_{1}+\frac{\partial \sigma_{12}}{\partial x_{2}}+\frac{\partial \sigma_{13}}{\partial x_{3}}=0, \\
i \operatorname{St} \sigma_{12}+\operatorname{HoT}_{\sigma_{12}}+\frac{\partial U_{1}}{\partial x_{2}}=-\delta_{\mathrm{rp}} \sigma_{12}+2 \int\left(L-L_{\mathrm{s}}\right) v_{1} v_{2} \mathrm{~d} \boldsymbol{v}, \\
i \mathrm{St} \sigma_{13}+\mathrm{HoT}_{\sigma_{13}}+\frac{\partial U_{1}}{\partial x_{3}}=-\delta_{\mathrm{rp}} \sigma_{13}+2 \int\left(L-L_{\mathrm{s}}\right) v_{1} v_{3} \mathrm{~d} \boldsymbol{v},
\end{array}
$$

which leads to the following diffusion-type equation for the flow velocity $U_{1}$ that is solved in a stable iterative manner:

$$
2 i \operatorname{St}\left(i \operatorname{St}+\bar{\delta}_{\mathrm{rp}}\right) U_{1}^{(k+1)}-\left(\frac{\partial^{2}}{\partial x_{2}^{2}}+\frac{\partial^{2}}{\partial x_{3}^{2}}\right) U_{1}^{(k+1)}=\operatorname{Source}+2 i \operatorname{St}\left(\bar{\delta}_{\mathrm{rp}}-\delta_{\mathrm{rp}}\right) U_{1}^{(k+1 / 2)},
$$


where $\bar{\delta}_{\mathrm{rp}}$ is given in Eq. (52), and

$$
\begin{aligned}
\text { Source }= & \frac{\partial}{\partial x_{2}}\left[2 \int\left(L^{(k)}-L_{\mathrm{s}}^{(k)}\right) v_{1} v_{2} \mathrm{~d} \boldsymbol{v}-\mathrm{HoT}_{\sigma_{12}}^{(k+1 / 2)}\right] \\
& +\frac{\partial}{\partial x_{3}}\left[2 \int\left(L^{(k)}-L_{\mathrm{s}}^{(k)}\right) v_{1} v_{3} \mathrm{~d} \boldsymbol{v}-\mathrm{HoT}_{\sigma_{13}}^{(k+1 / 2)}\right] .
\end{aligned}
$$

In numerical simulations, the molecular velocities $v_{2}$ and $v_{3}$ are discretized non-uniformly according to Eq. (42) with 48 points in each direction, while $v_{1}$ is truncated into the region of $[-6,6]$ and discretized by 24 uniformly-distributed points. Due to the symmetry $h\left(x_{2}, x_{3}, v_{1}, v_{2}, v_{3}\right)=h\left(x_{2}\right.$, Asp $\left.-x_{3}, v_{1}, v_{2},-v 3\right)$, we only consider the domain where $0 \leq x_{2} \leq 1$ and $0 \leq x_{3} \leq \mathrm{Asp} / 2$, which are discretized by 50 and 60 points according to Eq. (44) in $x_{1}$ and $x_{2}$ directions, respectively. The velocity distribution function entering the domain from the stationary walls is zero, while that from the oscillating wall is $2 v_{1} f_{\text {eq }}$. The diffusion-type equation (65) is approximated by the central finite difference with 5 stencils, which are directly solved by rewriting it in matrix form. From the inset of Fig. 16.(b) we see that the GSIS is very efficient as converged solutions are obtained within 40 iterations.

We are interested in how the average shear force exerting on the oscillating lid change with the normalized oscillation frequency St. Hence $\sigma_{13}$ is not considered here as it is antisymmetric along the line $x_{3}=\mathrm{Asp} / 2$ so its overall contribution to the friction is zero. The amplitude of the average shear force on the oscillating lid is defined as

$$
\bar{\sigma}_{12}=\frac{2\left|\int_{0}^{\mathrm{Asp} / 2} \sigma_{12}\left(x_{2}=1\right) \mathrm{d} x_{3}\right|}{\operatorname{Asp}},
$$

which is shown in Fig. 16(b) for different aspect ratios of the cavity over a wide range of the oscillation frequency, when $\delta_{\mathrm{rp}}=50$. It can be seen when Asp $=2$, the average shear force is the same as that of Asp $=\infty$, except that it is slightly larger when St is small. This is seen more clearly in Fig. 16.(c) that the two lateral walls, i.e. the left and right walls in Fig. 16.(a), increase the shear stress from a nearly small constant to a high rise near the left top corner. When St increases, the shear stress quickly decays from the oscillating lid to the zero value at the bottom surface, and its value at the oscillating lid is nearly uniform, see Fig. 16.(d) and (e). As the aspect ratio of the cavity reduces, the average shear force increases when St is small, see Fig. 16(f) for an example; this is easy to understood as the lateral walls increase the total friction according to our daily life experience. However, from Fig. 16(f) and (g) we can see that the shear stress quickly saturates, as larger oscillation frequency only slightly increases the shear stress at the lid, such that the average shear stress on the lid remains nearly constant over a wide range of St; and the smaller the aspect ratio is, the wider this region is. This may be useful to design a micro-electro-mechanical system where the shear force remains constant in a certain wide range of oscillation frequency. It is this efficient algorithm allowing us to find this new phenomenon which is missed in Ref. [58]. Another counter-intuitive thing is that, when St is large, the average shear force at small values of cavity aspect ratio is slightly smaller than that of the one-dimensional cavity, although the relative difference is within $5 \%$. 

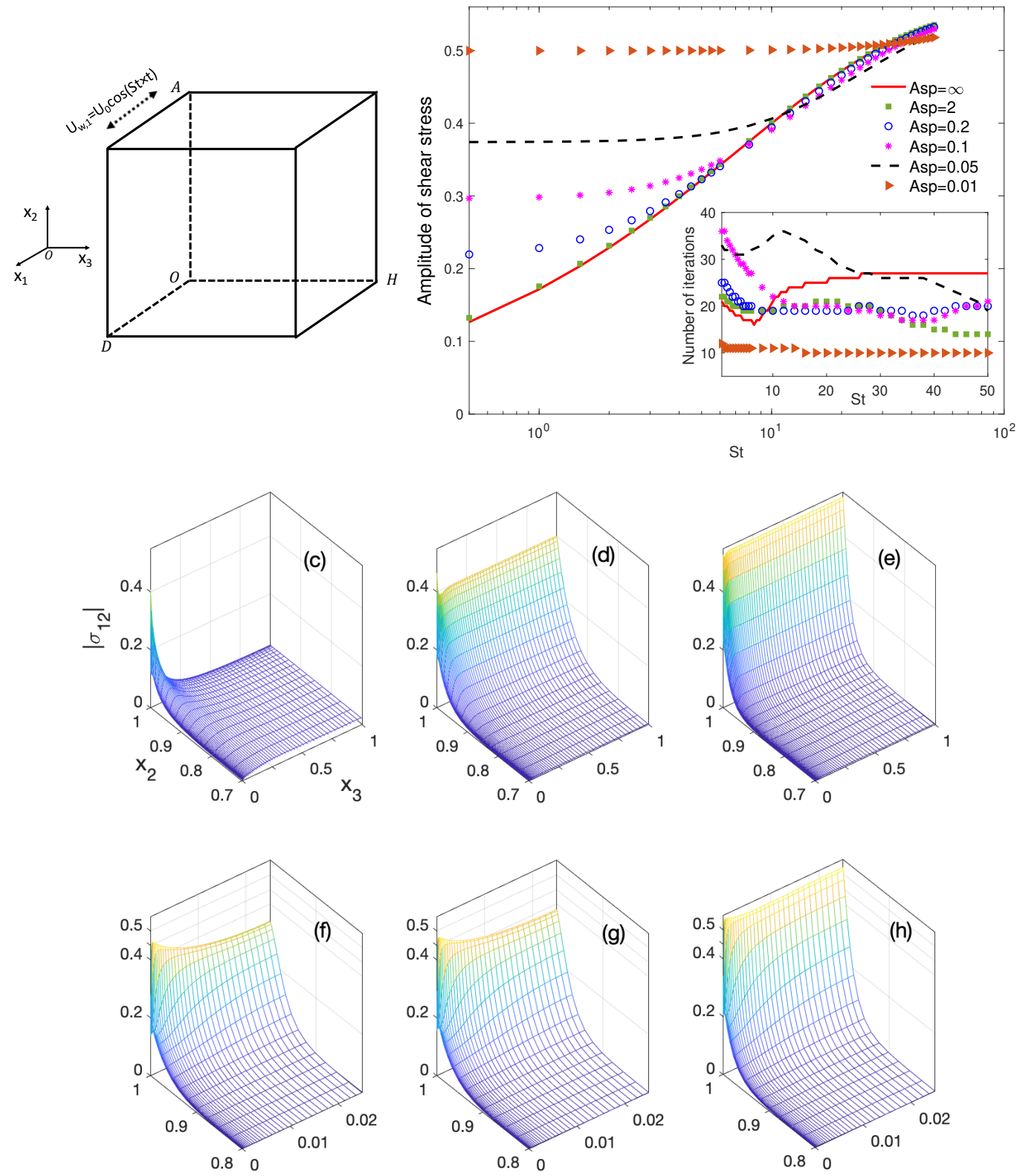

Figure 16: (a) Schematic of the oscillatory flow in a 3D rectangular cavity, where ' $\mathrm{O}$ ' is the origin of the coordinate. The top lid oscillates in the $x_{1}$ direction periodically. (b) The amplitude of shear force exerting on the oscillating lid that is normalized by the aspect ration Asp $=O H / O A$, see Eq. (67); Inset shows the iteration number when the relative error in $U_{1}$ between two consecutive iteration is less than $10^{-5}$. (c, d, e) The distribution of shear stress when $\mathrm{St}=0,10$, and 50, respectively, and Asp $=2$. (f, g, h) Same as (c, d, e), respectively, but with Asp $=0.05$. The linearized Shakhov model is used with $\delta_{\mathrm{rp}}=50$ in all cases. 


\section{Conclusions and outlooks}

In summary, we have developed a general synthetic iterative scheme to find the steadystate solution of the linearized Boltzmann equation efficiently and accurately. Various numerical results have demonstrated that our scheme is able to find the converged solution within about 20 iterations at any Knudsen number, due to the fact that the synthetic macroscopic equations not only asymptotically preserve the Navier-Stokes limit in the framework of Chapman-Enskog expansion 2 , but also explicitly contain the constitutive laws for the stress and heat flux at the first order approximation in the the Knudsen number to the linearized Boltzmann equation. As a consequence, accurate solutions that are not contaminated by numerical dissipation and accumulated error can be obtained when the cell size is much larger than the mean free path of gas molecules. Moreover, the numerical error in the general synthetic iterative scheme decays very fast and the convergence criterion can be set at a much higher value than the conventional iterative scheme. These factors enable our general synthetic iterative scheme to find the steady-state solution in 10-ish iterations.

This paper provides a framework to solve the general linear rarefied gas flow problems. The essence of our approach relies on the following two points: (i) the explicit inclusion of Navier-Stokes constitutive laws and (ii) high-order terms are derived exactly from the gas kinetic equation. The first point ensures fast convergence in the (near) continuum flow regime, while the latter ensures that correct solution is obtained in transition and freemolecular flow regimes. The advantages and future works are highlighted below:

1. Compared to the implicit UGKS [14] and it variants [45, 46], we conclude that in order to develop efficient multiscale numerical schemes, macroscopic equations must be solved together with the Boltzmann or kinetic model equations. While in Refs. 14, [45, 46] only five equations from the conservation laws are used so that complex flux evaluation across the cell interface must be adopted to asymptotically preserve the Navier-Stokes limit, our general synthetic iterative scheme needs no complex flux evaluation as the Navier-Stokes equations are recovered explicitly. Thus, the numerical implementation of GSIS is much easy than UGKS and the convergence to steady-state solution is much faster. More importantly, our scheme does not depend on the specific form of the collision operator, while that in Refs. [14, 45, 46] relies only on the BGK-type kinetic equations to enable exact evaluation of numerical flux.

2. The gas kinetic equation and synthetic equations can be solved by sophisticated methods of computational fluid dynamics. For highly rarefied gas flows, the cell size is easily smaller than the mean free path and both methods yield high accuracy. For continuum/or near continuum flows, as long as the macroscopic solver for synthetic

\footnotetext{
${ }^{2}$ If the numerical scheme solving the gas kinetic equation is not asymptotically preserving the NavierStokes limit, then the solution will suffer from huge numerical dissipation if the cell size is much larger than the mean free path; the numerical evidence in Fourier flow, oscillating Couette flow, sound propagation, and shear-driven flow between two eccentric cylinders has shown that in GSIS the cell size can be much larger than the mean free path to obtain accurate result, but the CIS cannot. However, the rigorous proof of the asymptotically preserving property of GSIS is very difficult, and we leave it to future works.
} 
equations (essentially the NS equations) is able to capture the continuum flow behaviors, the accuracy of GSIS is guaranteed, that is, the numerical cell size should be smaller than the variation scale (such as the wavelength of sound) of the flow, but can be much larger than the MFP. In other words, the solution of GSIS is not affected when gas kinetic equation and synthetic equations are discretized by different schemes, as long as synthetic equations capture the flow dynamics in the continuum regime. For example, in Fourier/oscillating Couette/sound propagation problems, the gas kinetic equation is solved by the second-order upwind finite difference scheme, while synthetic equations are solved by the central finite difference scheme.

3. Since the limitation on spatial cell size (i.e. it should be smaller than the mean free path of gas molecules) is removed and fast convergence is enabled, the present general synthetic iterative scheme may be applied to the low-variance [59, 60] and even frequency-domain [61] DSMC that solves the linearized Boltzmann and kinetic model equations to improve the computational efficiency, especially in the near-continuum flow regime.

4. The present method can be extended to multi-species and compressible flow. The key is to construct macroscopic equations that recover the compressible Navier-Stokes equation to the first order of Knudsen number. As a matter of fact, the Grad 13 moment equations [48, 49] can be directly used if the high-order velocity moments are calculated from the numerical solution of the Boltzmann equation, rather than closed by making assumption on the form of velocity distribution function. Actually the authors have implemented the general synthetic iterative scheme for nonlinear Fourier heat transfer between two parallel plates: started from the global equilibrium distribution, converged solution at arbitrary Knudsen number is found within 20 iterations.

5. It is noted that recently the unified gas-kinetic wave-particle (UGKWP) method, which uses the essential idea of UGKS that the streaming and collision should be treated spontaneously, has been applied in the framework of DSMC to remove the constraint on the cell size when the Knudsen number is small [62, 63]; the (ellipsoidal statistics) BGK kinetic model is solved and the complex and time-consuming particle sorting is used to enable the asymptotically preserving property. We believe that the general synthetic iterative scheme can also be applied to DSMC to remove the limitation on cell size and boost convergence, and the advantage is clear: it relies on no specific collision operator so that it can be extended naturally to multi-species flows and even flows involving chemical reactions.

With these new development implemented, it is foreseen that in the near future the problem of numerical simulation of multiscale rarefied gas flows may be solved completely. Also, the same idea can be applied to other kinetic equations such as the Enskog equation for dense gases dynamics with applications to gas extraction in unconventional reservoirs and non-equilibrium evaporation and condensation at the nano scale [64, 65, 66]. 


\section{Appendix}

Here, some details to solve the synthetic macroscopic equations using the high-order hybridizable discontinuous Galerkin (HDG) method [67] on arbitrary triangular mesh are presented. The steady-state governing equations can be written in the following mixed form as a system of first-order equations

$$
\begin{aligned}
& \nabla \cdot\left[\mathcal{G}_{\mathrm{c}}+\mathcal{G}_{\mathrm{d}}\right]=0, \\
& \boldsymbol{L}-\nabla \boldsymbol{u}-\boldsymbol{\Pi}=0, \\
& \boldsymbol{E}-\nabla T-\boldsymbol{\Theta}=0,
\end{aligned}
$$

where

$$
\begin{array}{r}
\mathcal{G}_{\mathrm{c}}=\left[\begin{array}{c}
\boldsymbol{U} \\
p \boldsymbol{I} \\
\mathbf{0}
\end{array}\right], \\
\boldsymbol{\mathbf { 0 }} \\
\boldsymbol{\Pi}=\left[\begin{array}{c}
\mathrm{\mathcal {G } _ { \mathrm { d } }}=\left[\begin{array}{c}
\mathrm{T} \\
-\frac{1}{\delta_{\mathrm{rp}}}\left(\boldsymbol{L}+\frac{2}{3} \operatorname{tr}(\boldsymbol{L}) \boldsymbol{I}\right) \\
-\frac{5}{4 \delta_{\mathrm{rp}} \operatorname{Pr}} \boldsymbol{E}
\end{array}\right], \\
\frac{1}{2} \operatorname{HoT}_{\sigma_{11}}+\frac{1}{2} \mathrm{HoT}_{\sigma_{22}} \\
\frac{1}{2} \mathrm{HoT}_{\sigma_{12}} \operatorname{HoT}_{\sigma_{11}}+\operatorname{HoT}_{\sigma_{22}}
\end{array}\right], \\
\boldsymbol{\Theta}=\left[\begin{array}{c}
\frac{4}{5} \mathrm{HoT}_{q_{1}} \\
\frac{4}{5} \mathrm{HoT}_{q_{2}}
\end{array}\right],
\end{array}
$$

with $\boldsymbol{I}$ being the identity matrix. The auxiliary variables $\boldsymbol{L}$ and $\boldsymbol{E}$ are introduced to approximate the combination of the velocity gradient $\nabla \boldsymbol{U}$, temperature gradient $\nabla T$ and the high-order moments. Then, the stress tensor and heat flux are evaluated as

$$
\sigma_{i j}=-\frac{1}{\delta_{\mathrm{rp}}}\left(L_{i j}+L_{j i}-\frac{2}{3} L_{k k} \delta_{i j}\right), \quad q_{i}=-\frac{5}{4 \delta_{\mathrm{rp}} \operatorname{Pr}} E_{i} .
$$

Let $\Delta \in \mathbb{R}^{2}$ be an two-dimensional domain with boundary $\partial \Delta$ in the $x_{1}-x_{2}$ plane. Then, $\Delta$ is partitioned in $N_{\mathrm{r}}$ disjoint regular triangles $\Delta_{r}: \Delta=\cup_{r}^{N_{\mathrm{e}}} \Delta_{r}$. The boundaries $\partial \Delta_{r}$ of the triangles define a group of $N_{\mathrm{f}}$ faces $\Gamma_{c}: \Gamma=\cup_{r}^{N_{\mathrm{e}}}\left\{\partial \Delta_{r}\right\}=\cup_{c}^{N_{\mathrm{f}}}\left\{\Gamma_{c}\right\}$. For HDG discretization, two types of discontinuous finite element approximation space, one for solutions within $\Delta_{r}$ and the other for traces of solution on $\Gamma_{c}$, are defined as

$$
\begin{gathered}
\mathfrak{V}=\left\{\varphi:\left.\varphi\right|_{\Delta_{i}} \in \mathfrak{P}^{\mathcal{K}}\left(\Delta_{r}\right), \forall \Delta_{i} \subset \Delta\right\}, \\
\mathfrak{W}=\left\{\psi:\left.\psi\right|_{\Gamma_{c}} \in \mathfrak{P}^{\mathcal{K}}\left(\Gamma_{c}\right), \forall \Gamma_{c} \subset \Gamma\right\},
\end{gathered}
$$

where $\mathfrak{P}^{\mathcal{K}}(D)$ denotes the space of $\mathcal{K}$-th order polynomials on a domain $D$.

The HDG method solves the system in two steps. First, a global problem is set up to determine the traces of the flow properties $\hat{\boldsymbol{Q}}=[\hat{p}, \hat{\boldsymbol{U}}, \hat{T}]$ on the faces $\Gamma$. Then, a local problem with $\hat{\boldsymbol{Q}}$ as the boundary condition on $\partial \Delta_{r}$ is solved element-by-element to obtain 
the solutions for the flow properties $\boldsymbol{Q}=[p, \boldsymbol{U}, T]$, as well as the ones for the auxiliary variables $\boldsymbol{L}$ and $\boldsymbol{E}$. Generally speaking, when moving from the interior of the triangle element $\Delta_{r}$ to its boundary $\partial \Delta_{r}$, the traces define what the values of field variables on the boundary should be. In the HDG method, it is assumed that the traces are singled-valued on each face.

We introduce the notations $(a, b)_{D}=\int_{D \in \mathbb{R}^{2}}(a \odot b) \mathrm{d} x_{1} \mathrm{~d} x_{2}$ and $\langle a, b\rangle_{D}=\int_{D \in \mathbb{R}^{1}}(a \odot b) \mathrm{d} \Gamma$, where $\odot$ can be either the dot product · or tensor product $\otimes$. The local problem is stated as: find $(\boldsymbol{Q}, \boldsymbol{L}, \boldsymbol{E}) \in[\mathfrak{V}]^{4} \times[\mathfrak{V}]^{4} \times[\mathfrak{V}]^{2}$ such that

$$
\begin{array}{r}
-\left(\mathcal{G}_{\mathrm{c}}+\mathcal{G}_{\mathrm{d}}, \nabla \boldsymbol{r}\right)_{\Delta_{r}}+\langle\hat{\mathcal{F}} \cdot \boldsymbol{n}, \boldsymbol{r}\rangle_{\partial \Delta_{r}}=0, \\
(\boldsymbol{L}, \boldsymbol{w})_{\Delta_{r}}+(\boldsymbol{U}, \nabla \cdot \boldsymbol{w})_{\Delta_{r}}-\langle\hat{\boldsymbol{U}}, \boldsymbol{w} \cdot \boldsymbol{n}\rangle_{\partial \Delta_{r}}=(\boldsymbol{w}, \boldsymbol{\Pi})_{\Delta_{r}}, \\
(\boldsymbol{E}, \boldsymbol{z})_{\Delta_{r}}+(T, \nabla \cdot \boldsymbol{z})_{\Delta_{r}}-\langle\hat{T}, \boldsymbol{z} \cdot \boldsymbol{n}\rangle_{\partial \Delta_{r}}=(\boldsymbol{z}, \boldsymbol{\Theta})_{\Delta_{r}},
\end{array}
$$

for all $(\boldsymbol{r}, \boldsymbol{w}, \boldsymbol{z}) \in[\mathfrak{V}]^{4} \times[\mathfrak{V}]^{4} \times[\mathfrak{V}]^{2}$. The numerical fluxes $\hat{\mathcal{F}} \cdot \boldsymbol{n}$ is defined as 68

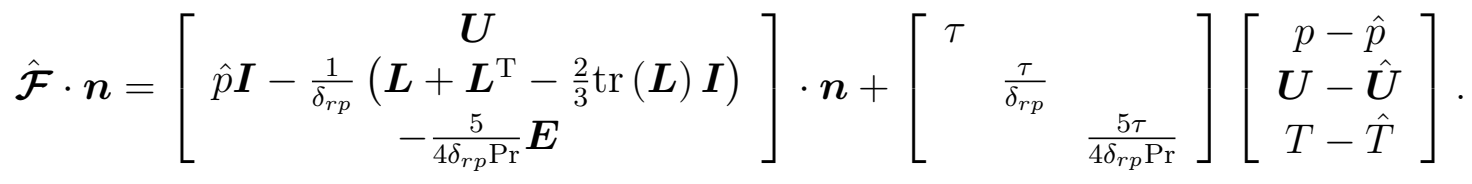

Here $\boldsymbol{n}$ being the outward unite normal vector of $\partial \Delta_{r}$. $\tau$ is the stabilization parameter that have important effects on the accuracy and convergence of the HDG method. In this work, we chosen $\tau=1 / H_{\min }$, with $H_{\min }$ the minimum height of the triangles.

The global problem is set up by enforcing the continuity of the numerical fluxes over all the interior faces. It is stated as: find $\hat{\boldsymbol{Q}} \in[\mathfrak{W}]^{4}$ such that

$$
\left\langle(\hat{\mathcal{F}} \cdot \boldsymbol{n})^{+}, \boldsymbol{\psi}\right\rangle_{\Gamma_{c}}+\left\langle(\hat{\mathcal{F}} \cdot \boldsymbol{n})^{-}, \boldsymbol{\psi}\right\rangle_{\Gamma_{c}}=0, \quad \text { on } \Gamma_{c} \in \Gamma \backslash \partial \Delta
$$

for all $\psi \in[\mathfrak{W}]^{4}$. Here the superscripts \pm denote the numerical fluxes obtained from the triangles on both sides of the face. Note that the traces on boundary faces are calculated as

$$
\left\langle\hat{\boldsymbol{Q}}-\boldsymbol{Q}_{\mathrm{VDF}}^{+}, \boldsymbol{\psi}\right\rangle_{\Gamma_{c}}, \quad \text { on } \Gamma_{c} \in \Gamma \cap \partial \Delta
$$

where $\boldsymbol{Q}_{\mathrm{VDF}}^{+}$is the field solutions directly calculated from the approximated velocity distribution function (see Eq. (8)) within the triangle where the boundary face $\Gamma_{c}$ belongs to.

By assembling the local problem (A.5) and global problem (A.7) and (A.8) over all the triangles and faces, we can obtain a matrix system of form

$$
\left[\begin{array}{llll}
A_{Q} & A_{L} & A_{E} & A_{\hat{Q}} \\
B_{Q} & B_{L} & B_{E} & B_{\hat{Q}} \\
C_{Q} & C_{L} & C_{E} & C_{\hat{Q}} \\
D_{Q} & D_{L} & D_{E} & D_{\hat{Q}}
\end{array}\right]\left[\begin{array}{c}
\mathbb{Q} \\
\mathbb{L} \\
\mathbb{E} \\
\hat{\mathbb{Q}}
\end{array}\right]=\left[\begin{array}{c}
S_{Q} \\
S_{L} \\
S_{E} \\
S_{\hat{Q}}
\end{array}\right]
$$


where $\mathbb{Q}, \mathbb{L}, \mathbb{E}$ and $\hat{\mathbb{Q}}$ are the vectors of degrees of freedom of the flow properties $\boldsymbol{Q}$, the auxiliary variables $\boldsymbol{L}$ and $\boldsymbol{E}$, and the traces of the flow properties $\hat{\boldsymbol{Q}}$, respectively. Note that the degrees of freedom for $\boldsymbol{Q}, \boldsymbol{L}$ and $\boldsymbol{E}$ are grouped together and ordered element-byelement, and the corresponding coefficient matrix $\left[A_{Q}, A_{L}, A_{E} ; B_{Q}, B_{L}, B_{E} ; C_{Q}, C_{L}, C_{E}\right]$ has block-diagonal structure. Therefore, we can eliminate $\boldsymbol{Q}, \boldsymbol{L}$ and $\boldsymbol{E}$ to obtained a reduced linear system involving only $\hat{\mathbb{Q}}$. Once $\hat{\mathbb{Q}}$ is determined, $\boldsymbol{Q}, \boldsymbol{L}$ and $\boldsymbol{E}$ are reconstructed corresponding to the local problem $\mathrm{A} .5$ in an element-wise fashion, while the stress tensor and heat flux are calculated as Eq. A.3.

\section{Acknowledgments}

This work is supported in the UK by the Engineering and Physical Sciences Research Council under grant EP/R041938/1. L. Zhu acknowledges the financial support of European Union's Horizon 2020 Research and Innovation Programme under the Marie SkłodowskaCurie grant agreement number 793007.

\section{References}

[1] S. Chapman, T. G. Cowling, The Mathematical Theory of Non-uniform Gases, Cambridge University Press, 1970.

[2] L. Wu, On the accuracy of macroscopic equations in the dynamic light scattering by rarefied gas, ResearchGate.

[3] G. A. Bird, Direct simulation and the Boltzmann equation, Physics of Fluids 13 (11) (1970) 2676-2681.

[4] V. V. Aristov, Direct Methods for Solving the Boltzmann Equation and Study of Nonequilibrium Flows, Springer Netherlands, Dordrecht, 2001.

[5] J. M. Burt, I. D. Boyd, A low diffusion particle method for simulating compressible inviscid flows, Journal of Computational Physics 227 (9) (2008) 4653-4670.

[6] L. Wu, J. Zhang, H. Liu, Y. Zhang, J. M. Reese, A fast iterative scheme for the linearized Boltzmann equation, Journal of Computational Physics 338 (2017) 431-451.

[7] L. Mieussens, Discrete-velocity models and numerical schemes for the Boltzmann-BGK equation in plane and axisymmetric geometries, Journal of Computational Physics 162 (2) (2000) 429-466.

[8] F. Tcheremissine, Direct numerical solution of the Boltzmann equation, AIP Conference Proceedings 762 (1) (2005) 677-685.

[9] C. Mouhot, L. Pareschi, Fast algorithms for computing the Boltzmann collision operator, Mathematics of Computation 75 (2006) 1833-1852.

[10] K. Xu, J.-C. Huang, An improved unified gas-kinetic scheme and the study of shock structures, IMA Journal of Applied Mathematics 76 (5) (2011) 698-711.

[11] J.-C. Huang, K. Xu, P. Yu, A unified gas-kinetic scheme for continuum and rarefied flows II: Multidimensional cases, Communications in Computational Physics 12 (3) (2012) 662-690.

[12] Z. Guo, K. Xu, R. Wang, Discrete unified gas kinetic scheme for all Knudsen number flows: Low-speed isothermal case, Physical Review E 88 (2013) 033305.

[13] Z. Guo, R. Wang, K. Xu, Discrete unified gas kinetic scheme for all Knudsen number flows. II. thermal compressible case, Physical Review E 91 (2015) 033313.

[14] Y. Zhu, C. Zhong, K. Xu, Implicit unified gas-kinetic scheme for steady state solutions in all flow regimes, Journal of Computational Physics 315 (2016) 16-38.

[15] S. Chen, K. Xu, A comparative study of an asymptotic preserving scheme and unified gas-kinetic scheme in continuum flow limit, Journal of Computational Physics 288 (2015) 52-65.

[16] P. Wang, M. T. Ho, L. Wu, Z. Guo, Y. Zhang, A comparative study of discrete velocity methods for low-speed rarefied gas flows, Computers \& Fluids 161 (2018) 33-46. 
[17] R. Roveda, D. B. Goldstein, P. L. Varghese, Hybrid Euler/particle approach for continuum/rarefied flows, Journal of Spacecraft and Rockets 35 (3) (1998) 258-265.

[18] W.-L. Wang, I. Boyd, Hybrid DSMC-CFD simulations of hypersonic flow over sharp and blunted bodies, in: 36th AIAA Thermophysics Conference, 2003, pp. 1-13.

[19] Q. Sun, I. D. Boyd, G. V. Candler, A hybrid continuum/particle approach for micro-scale gas flows, in: Rarefied Gas Dyanmics: 23rd International Symposium, 2003, pp. 752-759.

[20] H. S. Wijesinghe, R. D. Hornung, A. L. Garcia, N. G. Hadjiconstantinou, Three-dimensional hybrid continuum-atomistic simulations for multiscale hydrodynamics, Journal of Fluids Engineering $126(5)$ (2004) 768-777.

[21] T. Schwartzentruber, L. Scalabrin, I. Boyd, A modular particle-continuum numerical method for hypersonic non-equilibrium gas flows, Journal of Computational Physics 225 (1) (2007) 1159-1174.

[22] V. Kolobov, R. Arslanbekov, V. Aristov, A. Frolova, S. Zabelok, Unified solver for rarefied and continuum flows with adaptive mesh and algorithm refinement, Journal of Computational Physics 223 (2) (2007) 589-608.

[23] Z. Tang, B. He, G. Cai, Investigation on a coupled Navier-Stokes-Direct simulation Monte Carlo method for the simulation of plume flowfield of a conical nozzle, International Journal for Numerical Methods in Fluids 76 (2) (2014) 95-108.

[24] M. L. Adams, E. W. Larsen, Fast iterative methods for discrete-ordinates particle transport calculations, Progress in Nuclear Energy 40 (1) (2002) 3-159.

[25] D. Valougeorgis, S. Naris, Acceleration schemes of the discrete velocity method: Gaseous flows in rectangular microchannels, SIAM Journal on Scientific Computing 25 (2) (2003) 534-552.

[26] J. Lihnaropoulos, S. Naris, D. Valougeorgis, Formulation and stability analysis of rapidly convergent iteration schemes for the 2-D linearized BGK equation, Transport Theory and Statistical Physics 36 (46) (2007) 513-528.

[27] L. Szalmás, D. Valougeorgis, A fast iterative model for discrete velocity calculations on triangular grids, Journal of Computational Physics 229 (11) (2010) 4315-4326.

[28] S. Naris, D. Valougeorgis, F. Sharipov, D. Kalempa, Discrete velocity modelling of gaseous mixture flows in MEMS, Superlattices and Microstructures 35 (3) (2004) 629-643.

[29] S. Naris, D. Valougeorgis, D. Kalempa, F. Sharipov, Gaseous mixture flow between two parallel plates in the whole range of the gas rarefaction, Physica A: Statistical Mechanics and its Applications 336 (3) (2004) 294-318.

[30] S. Naris, D. Valougeorgis, D. Kalempa, F. Sharipov, Flow of gaseous mixtures through rectangular microchannels driven by pressure, temperature, and concentration gradients, Physics of Fluids 17 (10) (2005) 100607.

[31] L. Szalmás, Accelerated discrete velocity method for axial-symmetric flows of gaseous mixtures as defined by the McCormack kinetic model, Computer Physics Communications 184 (11) (2013) 24302437.

[32] L. Szalmás, An accelerated discrete velocity method for flows of rarefied ternary gas mixtures in long rectangular channels, Computers \& Fluids 128 (2016) 91-97.

[33] W. Su, P. Wang, H. Liu, L. Wu, Accurate and efficient computation of the Boltzmann equation for Couette flow: Influence of intermolecular potentials on Knudsen layer function and viscous slip coefficient, Journal of Computational Physics 378 (2019) 573-590.

[34] J. Fan, C. Shen, Statistical simulation of low-speed rarefied gas flows, Journal of Computational Physics 167 (2) (2001) 393-412.

[35] J. Zhang, J. Fan, J. Jiang, Multiple temperature model for the information preservation method and its application to nonequilibrium gas flows, Journal of Computational Physics 230 (19) (2011) 7250-7265.

[36] F. Fei, J. Fan, A diffusive information preservation method for small Knudsen number flows, Journal of Computational Physics 243 (2013) 179-193.

[37] P. Degond, G. Dimarco, L. Pareschi, The moment-guided Monte Carlo method, International Journal for Numerical Methods in Fluids 67 (2) (2011) 189-213.

[38] W. T. Taitano, D. A. Knoll, L. Chacón, J. M. Reisner, A. K. Prinja, Moment-based acceleration for 
neutral gas kinetics with BGK collision operator, Journal of Computational and Theoretical Transport $43(1-7)$ (2014) 83-108.

[39] L. Wu, J. M. Reese, Y. Zhang, Solving the Boltzmann equation deterministically by the fast spectral method: application to gas microflows, Journal of Fluid Mechanics 746 (2014) 53-84.

[40] L. Wu, C. White, T. J. Scanlon, J. M. Reese, Y. Zhang, Deterministic numerical solutions of the Boltzmann equation using the fast spectral method, Journal of Computational Physics 250 (2013) 27-52.

[41] L. Wu, H. Liu, Y. Zhang, J. M. Reese, Influence of intermolecular potentials on rarefied gas flows: Fast spectral solutions of the Boltzmann equation, Physics of Fluids 27 (8) (2015) 082002.

[42] T. Ohwada, Y. Sone, K. Aoki, Numerical analysis of the shear and thermal creep flows of a rarefied gas over a plane wall on the basis of the linearized Boltzmann equation for hard-sphere molecules, Physics of Fluids A: Fluid Dynamics 1 (9) (1989) 1588-1599.

[43] W. Su, P. Wang, Y. Zhang, L. Wu, A high-order hybridizable discontinuous Galerkin method with fast convergence to steady-state solutions of the gas kinetic equation, Journal of Computational Physics 376 (2019) 973-991.

[44] W. Su, P. Wang, Y. Zhang, L. Wu, Implicit discontinuous Galerkin method for the Boltzmann equation (2019) arXiv:1901.01865.

[45] L. M. Yang, C. Shu, W. M. Yang, Z. Chen, H. Dong, An improved discrete velocity method (DVM) for efficient simulation of flows in all flow regimes, Physics of Fluids 30 (6) (2018) 062005.

[46] L. M. Yang, Z. Chen, C. Shu, W. M. Yang, J. Wu, L. Q. Zhang, Improved fully implicit discrete-velocity method for efficient simulation of flows in all flow regimes, Physical Review E 98 (2018) 063313.

[47] E. M. Shakhov, Approximate kinetic equations in rarefied gas theory, Fluid Dynamics 3 (1) (1968) $112-115$.

[48] H. Grad, On the kinetic theory of rarefied gases, Communications on Pure and Applied Mathematics 2 (4) (1949) 331-407.

[49] H. Struchtrup, Macroscopic Transport Equations for Rarefied Gas Fows: Approximation Methods in Kinetic Theory, Heidelberg, Germany: Springer, 2005.

[50] F. Sharipov, D. Kalempa, Oscillatory Couette flow at arbitrary oscillation frequency over the whole range of the Knudsen number, Microfluidics and Nanofluidics 4 (5) (2008) 363-374.

[51] D. Kalempa, F. Sharipov, Sound propagation through a rarefied gas confined between source and receptor at arbitrary Knudsen number and sound frequency, Physics of Fluids 21 (10) (2009) 103601.

[52] L. Wu, J. Reese, Y. Zhang, Oscillatory rarefied gas flow inside rectangular cavities, Journal of Fluid Mechanics 748 (2014) 350-367.

[53] A. Sugawara, S. Yip, L. Sirovich, Spectrum of density fluctuations in gases, The Physics of Fluids 11 (5) (1968) 925-932.

[54] L. Wu, C. White, T. J. Scanlon, J. M. Reese, Y. Zhang, A kinetic model of the Boltzmann equation for non-vibrating polyatomic gases, Journal of Fluid Mechanics 763 (2015) 24-50.

[55] W. Su, S. Lindsay, H. Liu, L. Wu, Comparative study of the discrete velocity and lattice Boltzmann methods for rarefied gas flows through irregular channels, Physical Review E 96 (2017) 023309.

[56] F. Sharipov, Data on the velocity slip and temperature jump on a gas-solid interface, Journal of Physical and Chemical Reference Data 40 (2) (2011) 023101.

[57] O. Schenk, K. Gärtner, Solving unsymmetric sparse systems of linear equations with PARDISO, Future Generation Computer Systems 20 (3) (2004) 475 - 487, selected numerical algorithms.

[58] P. Wang, W. Su, Y. Zhang, Oscillatory rarefied gas flow inside a three dimensional rectangular cavity, Physics of Fluids 30 (10) (2018) 102002.

[59] G. A. Radtke, N. G. Hadjiconstantinou, Variance-reduced particle simulation of the Boltzmann transport equation in the relaxation-time approximation, Physical Review E 79 (2009) 056711.

[60] G. A. Radtke, N. G. Hadjiconstantinou, W. Wagner, Low-noise Monte Carlo simulation of the variable hard sphere gas, Physics of Fluids 23 (3) (2011) 030606.

[61] D. R. Ladiges, J. E. Sader, Frequency-domain Monte Carlo method for linear oscillatory gas flows, Journal of Computational Physics 284 (2015) 351-366. 
[62] C. Liu, Y. Zhu, K. Xu, Unified gas-kinetic wave-particle methods I: Continuum and rarefied gas flow, Journal of Computational Physics 401 (2020) 108977.

[63] Y. Zhu, C. Liu, C. Zhong, K. Xu, Unified gas-kinetic wave-particle methods. II. multiscale simulation on unstructured mesh, Physics of Fluids 31 (6) (2019) 067105.

[64] L. Wu, Y. Zhang, J. M. Reese, Fast spectral solution of the generalized Enskog equation for dense gases, Journal of Computational Physics 303 (2015) 66-79.

[65] L. Wu, H. Liu, J. M. Reese, Y. Zhang, Non-equilibrium dynamics of dense gas under tight confinement, Journal of Fluid Mechanics 794 (2016) 252-266.

[66] A. Frezzotti, L. Gibelli, S. Lorenzani, Mean field kinetic theory description of evaporation of a fluid into vacuum, Physics of Fluids 17 (1) (2005) 012102.

[67] B. Cockburn, N. C. Nguyen, J. Peraire, A comparison of HDG methods for stokes flow, Journal of Scientific Computing 45 (1) (2010) 215-237.

[68] J. Peraire, N. Nguyen, B. Cockburn, A hybridizable discontinuous Galerkin method for the compressible Euler and Navier-Stokes equations, in: 48th AIAA Aerospace Sciences Meeting Including the New Horizons Forum and Aerospace Exposition, 2010, pp. 1-11. 